\title{
Multi-strain virus dynamics with mutations: A global analysis
}

\author{
Patrick De Leenheer*and Sergei S. Pilyugin ${ }^{\dagger}$ \\ Department of Mathematics \\ University of Florida, Gainesville, FL 32611-8105, USA
}

To our mentor and good friend Hal Smith, on the occasion of his 60th birthday.

\begin{abstract}
We consider within-host virus models with $n \geq 2$ strains and allow mutation between the strains. If there is no mutation, a Lyapunov function establishes global stability of the steady state corresponding to the fittest strain. For small perturbations this steady state persists, perhaps with small concentrations of some or all other strains, depending on the connectivity of the graph describing all possible mutations. Moreover, using a perturbation result due to Smith and Waltman [14, we show that this steady state also preserves global stability.
\end{abstract}

\section{Introduction}

The study of the dynamics of within-host virus disease models has been a very fruitful area of research over the past few decades. Of particular importance has been the work on mathematical models of HIV infection by Perelson and coauthors [10, 11] and Nowak and coauthors [9]. It has spurred more recent research by among others Hal Smith with one of us [1, [16] and [12.

For single-strain virus models, the understanding of the global behavior has been largely based on the fact that they are competitive [1] and the use of particular mathematical tools developed by Muldowney; see Li and Muldowney 8 for an application of these techniques to the classical SEIR model in epidemiology. Of course it is well known that for globally stable systems there is a Lyapunov function, but finding such a function is often difficult, as illustrated by the following quote from Smith and Waltman's classical work on chemostats [13] on p. 37:

\section{Considerable ingenuity, intuition, and perhaps luck are required to find a Liapunov function.}

One of the purposes of this paper is to find such Lyapunov functions for various within host virus models following the ingenuity from [6] and [5]. Another purpose of the paper is to investigate what happens if we include mutation effects in the model by allowing different virus strains to mutate into each other. This is very relevant in the context of HIV where mutations have profound impact on treatment, see for instance [12] where a two-strain model is considered.

Mathematically we will treat the model with mutations as a perturbation of the original model. It turns out that the structural properties of the mutation matrix that describes the possible mutations (in particular, whether this matrix is irreducible or not), dictate which single strain steady states of the unperturbed model persist in the perturbed model, and which don't. An obvious problem is to determine if the globally stable single strain steady state of the unperturbed model persists. We will show that this is always the case, regardless of the mutation matrix. Moreover, taking advantage of the perturbation result developed by Smith and Waltman in [14, we will show that this steady state remains globally stable for small values of the mutation parameter. In order to apply this perturbation result we will first need to establish a particular persistence property, uniform in the perturbation parameter, and to achieve this we invoke the theory developed by Hutson [3, 4, see also [2, which uses the notion of an average Lyapunov function. It will be shown that a rather simple -in fact, linear- average Lyapunov function exists.

\footnotetext{
*email: deleenhe@math.ufl.edu. Supported in part by NSF grant DMS-0614651.

$\dagger$ email: pilyugin@math.ufl.edu. Supported in part by NSF grant DMS-0517954.
} 
The paper is organized as follows. In Section 2 we present a Lyapunov function to establish global stability of the disease equilibrium of a single-strain virus model. This is extended in Section 3 to a global stability result for a multi-strain model which does not include mutations. In biological terms, we demonstrate that in the absence of mutations the fittest strain of the virus drives all other viral strains to extinction. In Section 4 we investigate what happens if mutations are taken into account for two different models. Finally, in the Appendix we extend all our results to a slightly modified model which includes an often neglected loss term in the virus equation.

\section{Single-strain}

In this paper, we consider the basic model of the form

$$
\begin{aligned}
\dot{T} & =f(T)-k V T \\
\dot{T}^{*} & =k V T-\beta T^{*} \\
\dot{V} & =N \beta T^{*}-\gamma V,
\end{aligned}
$$

where $T, T^{*}, V$ denote the concentrations of uninfected (healthy) and infected host cells, and free virions, respectively. Equations (11) describe a general viral infection where the viral replication is limited by the availability of target cells $T$. In this model, we assume that all infected cells $T^{*}$ are virus-producing cells, that is, we do not include any intermediate stage(s) corresponding to latently infected cells. In addition, we do not explicitly consider the impact of the immune response. Implicitly, the immune response can be accounted for by the removal term $-\beta T^{*}$. The rate of viral production is assumed proportional to the removal of infected cells. In case of lytic viruses, $N$ represents the average burst size of a single infected cell; whereas in case of budding viruses, $N$ can be thought of as the average number of virions produced over a lifetime of an infected cell. For different infections, the actual class of the target cells in (11) may vary from the $C D 4+T$ lymphocytes (in case of HIV), to the epithelial cells (in case of Influenza), to the red blood cells (in case of Malaria). The $T, T^{*}, V$ notation is adopted from the classical HIV model [11.

All parameters are assumed to be positive. The parameters $\beta$ and $\gamma$ are the removal rates of the infected cells and virus particles respectively. Following [11, 9], we neglect the term in the $V$-equation that represents the loss of a virus particle upon infection. But all subsequent results hold when this loss term is included, in which case the $V$-equation reads:

$$
\dot{V}=N \beta T^{*}-\gamma V-k V T \text {. }
$$

These results will be presented in the Appendix.

The growth rate of the uninfected cell population is given by the smooth function $f(T): \mathbb{R}_{+} \rightarrow \mathbb{R}$, which is assumed to satisfy the following:

$$
\exists T_{0}>0: \quad f(T)\left(T-T_{0}\right)<0, \quad T \neq T_{0} .
$$

Since continuity of $f$ implies that $f\left(T_{0}\right)=0$, it is easy to see that

$$
E_{0}=\left(T_{0}, 0,0\right)
$$

is an equilibrium of (11). Effectively, $T_{0}$ is the carrying capacity for the healthy cell population.

A second, positive equilibrium may exist if the following quantities are positive:

$$
\bar{T}=\frac{\gamma}{k N}, \quad \bar{T}^{*}=\frac{f(\bar{T})}{\beta}, \quad \bar{V}=\frac{f(\bar{T})}{k \bar{T}} .
$$

Note that this is the case if and only if $f\left(\frac{\gamma}{k N}\right)>0$, or equivalently by (2) that $\bar{T}=\frac{\gamma}{k N}<T_{0}$. In terms of the basic reproduction number

$$
\mathcal{R}^{0}:=\frac{k N}{\gamma} T_{0}=\frac{T_{0}}{\bar{T}_{0}}
$$

existence of a positive equilibrium is therefore equivalent to $\mathcal{R}^{0}>1$. We assume henceforth that $\mathcal{R}^{0}>1$ and denote the disease steady state by $E=\left(\bar{T}, \bar{T}^{*}, \bar{V}\right)$. Let us introduce the following sector condition:

$$
\text { (C) }(f(T)-f(\bar{T}))\left(1-\frac{\bar{T}}{T}\right) \leq 0 \text {. }
$$


Note that this condition is satisfied when $f(T)$ is a decreasing function, independently of the value of $\bar{T}$. For instance, 9] considers $f(T)=c_{1}-c_{2} T$, where $c_{i}$ are positive constants. Another example [1] is $f(T)=s+r T\left(1-\frac{T}{K}\right)$ provided that $f(0)=s \geq f(\bar{T})$.

Theorem 1. Let (C) hold. Then the equilibrium $E$ is globally asymptotically stable for (1) with respect to initial conditions satisfying $T^{*}(0)+V(0)>0$.

Proof. Consider the following function on $\operatorname{int}\left(\mathbb{R}_{+}^{3}\right)$ :

$$
W=\int_{\bar{T}}^{T}\left(1-\frac{\bar{T}}{\tau}\right) d \tau+\int_{\bar{T}^{*}}^{T^{*}}\left(1-\frac{\bar{T}^{*}}{\tau}\right) d \tau+\frac{\beta}{N \beta} \int_{\bar{V}}^{V}\left(1-\frac{\bar{V}}{\tau}\right) d \tau
$$

Then

$$
\begin{aligned}
\dot{W} & =(f(T)-k V T)\left(1-\frac{\bar{T}}{T}\right)+\left(k V T-\beta T^{*}\right)\left(1-\frac{\bar{T}^{*}}{T^{*}}\right)+\frac{1}{N}\left(N \beta T^{*}-\gamma V\right)\left(1-\frac{\bar{V}}{V}\right) \\
& =f(T)\left(1-\frac{\bar{T}}{T}\right)+k V \bar{T}-k V T \frac{\bar{T}^{*}}{T^{*}}+\beta \bar{T}^{*}-\beta T^{*} \frac{\bar{V}}{V}-\frac{\gamma}{N} V+\frac{\gamma}{N} \bar{V}
\end{aligned}
$$

Since from (3) we have that $\beta \bar{T}^{*}=k \bar{V} \bar{T}=\frac{\gamma}{N} \bar{V}$, it follows that

$$
\begin{aligned}
\dot{W} & =f(T)\left(1-\frac{\bar{T}}{T}\right)+\beta \bar{T}^{*} \frac{V}{\bar{V}}-\beta \bar{T}^{*} \frac{\bar{T}^{*} V T}{T^{*} \bar{V} \bar{T}}+\beta \bar{T}^{*}-\beta \bar{T}^{*} \frac{\bar{V} T^{*}}{V \bar{T}^{*}}-\beta T^{*} \frac{V}{\bar{V}}+\beta T^{*} \\
& =(f(T)-f(\bar{T}))\left(1-\frac{\bar{T}}{T}\right)+\beta T^{*}\left(1-\frac{\bar{T}}{T}\right)+\beta \bar{T}^{*} \frac{V}{\bar{V}}-\beta \bar{T}^{*} \frac{\bar{T}^{*} V T}{T^{*} \bar{V} \bar{T}}+\beta \bar{T}^{*}-\beta \bar{T}^{*} \frac{\bar{V} T^{*}}{V \bar{T}^{*}}-\beta T^{*} \frac{V}{\bar{V}}+\beta T^{*} \\
& =(f(T)-f(\bar{T}))\left(1-\frac{\bar{T}}{T}\right)-\beta \bar{T}^{*}\left[\frac{\bar{T}}{T}+\frac{\bar{T}^{*} V T}{T^{*} \bar{V} \bar{T}}+\frac{\bar{V} T^{*}}{V \bar{T}^{*}}-3\right]
\end{aligned}
$$

The first term is non-positive by $(\mathbf{C})$. The second term is non-positive as well since the geometric mean of 3 non-negative numbers is not larger than the arithmetic mean of those numbers. Hence, $\dot{W} \leq 0$ in int $\left(\mathbb{R}_{+}^{3}\right)$, and the local stability of $E$ follows. Notice that $\dot{W}$ equals zero iff both the first term and the second term are zero, and using $(\mathbf{C})$, this happens at points where:

$$
\frac{\bar{T}}{T}=1 \text { and } \frac{\bar{T}^{*} V}{T^{*} \bar{V}}=1
$$

Then LaSalle's Invariance Principle [7] implies that all bounded solutions in int $\left(\mathbb{R}_{+}^{3}\right)$ converge to the largest invariant set in

$$
M=\left\{\left(T, T^{*}, V\right) \in \operatorname{int}\left(\mathbb{R}_{+}^{3}\right) \mid \frac{\bar{T}}{T}=1, \frac{\bar{T}^{*} V}{T^{*} \bar{V}}=1\right\} .
$$

Firstly, boundedness of all solutions follows from Lemma 1 which is proved later in a more general setting. Secondly, it is clear that the largest invariant set in $M$ is the singleton $\{E\}$. Finally, note that forward solutions starting on the boundary of $\mathbb{R}_{+}^{3}$ with either $T_{1}(0)$ or $V_{1}(0)$ positive, enter int $\left(\mathbb{R}_{+}^{3}\right)$ instantaneously. This concludes the proof.

\section{Competitive exclusion in a multi-strain model}

Let us now consider a multi-strain model:

$$
\begin{aligned}
\dot{T} & =f(T)-\sum_{i=1}^{n} k_{i} V_{i} T \\
\dot{T}_{i}^{*} & =k_{i} V_{i} T-\beta_{i} T_{i}^{*}, \quad i=1, \ldots, n \\
\dot{V}_{i} & =N_{i} \beta_{i} T_{i}^{*}-\gamma_{i} V_{i}, \quad i=1, \ldots, n
\end{aligned}
$$

where all parameters are positive. Similar calculations as in the single-strain model show there is a unique disease-free equilibrium $E_{0}=\left(T_{0}, 0,0\right)$. For each $i$, there is a corresponding single-strain equilibrium $E_{i}$ with positive $T, T_{i}^{*}$ and $V_{i}$ components and zero components otherwise if and only if

$$
1<\mathcal{R}_{i}^{0}
$$


Here, $\mathcal{R}_{i}^{0}$ is the basic reproduction number for strain $i$ which is defined by

$$
\mathcal{R}_{i}^{0}=\frac{T_{0}}{\bar{T}^{i}} .
$$

The positive components of $E_{i}$ are then given by

$$
\bar{T}^{i}=\frac{\gamma_{i}}{k_{i} N_{i}}, \quad \bar{T}_{i}^{*}=\frac{f\left(\bar{T}^{i}\right)}{\beta_{i}}, \quad \bar{V}_{i}=\frac{f\left(\bar{T}^{i}\right)}{k_{i} \bar{T}^{i}} .
$$

We assume that all $E_{i}$ exist and assume without loss of generality (by possibly reordering components) that

$$
\bar{T}^{1}<\bar{T}^{2} \leq \cdots \leq \bar{T}^{n-1} \leq \bar{T}^{n}<T_{0},
$$

or equivalently, that

$$
1<\mathcal{R}_{n}^{0} \leq \mathcal{R}_{n-1}^{0} \leq \cdots \leq \mathcal{R}_{2}^{0}<\mathcal{R}_{1}^{0}
$$

and will prove the following competitive exclusion principle. It asserts that the strain with the lowest target cell concentration at steady state (or equivalently, with highest basic reproduction number) will ultimately dominate, provided that such strain is present initially.

Theorem 2. Assume that all $E_{i}$ exist for (4) - (6), that (C) holds with $\bar{T}^{1}$ instead of $\bar{T}$, and that (8) holds. Then $E_{1}$ is globally asymptotically stable for (4) - (6) with respect to initial conditions satisfying $T_{1}^{*}(0)+V_{1}(0)>0$.

Proof. Consider the following function on $U:=\left\{\left(T, T_{1}^{*}, \ldots, T_{n}^{*}, V_{1}, \ldots, V_{n}\right) \in \mathbb{R}^{2 n+1} \mid T, T_{1}^{*}, V_{1}>0\right\}$ :

$$
W=\int_{\bar{T}^{1}}^{T}\left(1-\frac{\bar{T}^{1}}{\tau}\right) d \tau+\int_{\bar{T}_{1}^{*}}^{T_{1}^{*}}\left(1-\frac{\bar{T}_{1}^{*}}{\tau}\right) d \tau+\frac{1}{N_{1}} \int_{\bar{V}_{1}}^{V_{1}}\left(1-\frac{\bar{V}_{1}}{\tau}\right) d \tau+\sum_{i=2}^{n}\left(T_{i}^{*}+\frac{1}{N_{i}} V_{i}\right) .
$$

Then

$$
\begin{aligned}
\dot{W}= & \left(f(T)-\sum_{i=1}^{n} k_{i} V_{i} T\right)\left(1-\frac{\bar{T}^{1}}{T}\right)+\left(k_{1} V_{1} T-\beta_{1} T_{1}^{*}\right)\left(1-\frac{\bar{T}_{1}^{*}}{T_{1}^{*}}\right)+\frac{1}{N_{1}}\left(N_{1} \beta_{1} T_{1}^{*}-\gamma_{1} V_{1}\right)\left(1-\frac{\bar{V}_{1}}{V_{1}}\right) \\
& +\sum_{i=2}^{n}\left(k_{i} V_{i} T-\frac{\gamma_{i}}{N_{i}} V_{i}\right) \\
= & \left(f(T)-k_{1} V_{1} T\right)\left(1-\frac{\bar{T}^{1}}{T}\right)+\left(k_{1} V_{1} T-\beta_{1} T_{1}^{*}\right)\left(1-\frac{\bar{T}_{1}^{*}}{T_{1}^{*}}\right)+\frac{1}{N_{1}}\left(N_{1} \beta_{1} T_{1}^{*}-\gamma_{1} V_{1}\right)\left(1-\frac{\bar{V}_{1}}{V_{1}}\right) \\
& -\sum_{i=2}^{n}\left(-k_{i} V_{i} \bar{T}^{1}+\frac{\gamma_{i}}{N_{i}} V_{i}\right)
\end{aligned}
$$

Notice that the first three terms can be simplified in a way similar as in the proof of Theorem 1, and using the expression for $\bar{T}^{i}$ in (7), we find that

$$
\begin{aligned}
\dot{W}= & \left(f(T)-f\left(\bar{T}^{1}\right)\right)\left(1-\frac{\bar{T}^{1}}{T}\right)-\beta_{1} \bar{T}_{1}^{*}\left[\frac{\bar{T}^{1}}{T}+\frac{\bar{T}_{1}^{*} V_{1} T}{T_{1}^{*} \bar{V}_{1} \bar{T}^{1}}+\frac{\bar{V}_{1} T_{1}^{*}}{V_{1} \bar{T}_{1}^{*}}-3\right] \\
& -\sum_{i=2}^{n} k_{i} V_{i}\left(\bar{T}^{i}-\bar{T}^{1}\right)
\end{aligned}
$$

Each of the first two terms is non-positive as was shown in the proof of Theorem 1 . The third part is also non-positive by (8). Thus $\dot{W} \leq 0$, establishing already stability of $E_{1}$. An application of LaSalle's Invariance Principle shows that all bounded solutions in $U$ (and as before, boundedness follows from Lemma 1 which is proved later) converge to the largest invariant set in

$$
\left\{\left(T, T_{1}^{*}, \ldots, T_{n}^{*}, V_{1}, \ldots, V_{n}\right) \in U \mid \frac{\bar{T}^{1}}{T}=1, \frac{\bar{T}_{1}^{*} V_{1}}{T_{1}^{*} \bar{V}_{1}}=1, \quad V_{i}=0, i>2\right\},
$$

which is easily shown to be the singleton $\left\{E_{1}\right\}$. Finally, solutions on the boundary of $U$ with $T_{1}^{*}(0)+V_{1}(0)>0$ enter $U$ instantaneously, which concludes the proof. 


\section{Perturbations by mutations}

In this section we expand model (44) - (6) to account for mutations between the $n$ strains. In fact, we will study two different ways in which mutations occur. Our first extended model can be written compactly as follows:

$$
\begin{aligned}
\dot{T} & =f(T)-k^{\prime} V T, \quad T \in \mathbb{R}_{+} \\
\dot{T}^{*} & =P(\mu) K V T-B T^{*}, \quad T^{*} \in \mathbb{R}_{+}^{n} \\
\dot{V} & =\hat{N} B T^{*}-\Gamma V, \quad V \in \mathbb{R}_{+}^{n},
\end{aligned}
$$

while the second is written as

$$
\begin{aligned}
\dot{T} & =f(T)-k^{\prime} V T, \quad T \in \mathbb{R}_{+} \\
\dot{T}^{*} & =K V T-B T^{*}, \quad T^{*} \in \mathbb{R}_{+}^{n} \\
\dot{V} & =P(\mu) \hat{N} B T^{*}-\Gamma V, \quad V \in \mathbb{R}_{+}^{n},
\end{aligned}
$$

In both models $K=\operatorname{diag}(k), B=\operatorname{diag}(\beta), \hat{N}=\operatorname{diag}(N)$ and $\Gamma=\operatorname{diag}(\gamma)$, and the matrix $P(\mu)$ with $\mu \in[0,1]$ is defined as follows:

$$
P(\mu)=I+\mu Q,
$$

where $Q$ is a matrix with $q_{i j}>0$ if strain $j$ can mutate to $i$ (for $i \neq j$ ) so that different magnitudes of $q_{i j}$ reflect the possible differences in the specific mutation rates. The diagonal entries of $Q$ are such that each column of $Q$ sums to zero. Notice that $P$ is a stochastic matrix (all its entries are in $[0,1]$ and all its columns sum to one) provided that $\mu \leq-1 / q_{i i}$ for all $i$ (which is assumed henceforth), and that $P(0)=I$.

Lemma 1. Both system (10) - (12) and (13) - (15) are dissipative, i.e. there exists a forward invariant compact set $K \subset \mathbb{R}_{+}^{2 n+1}$ such that every solution eventually enters $K$.

Proof. From (10) and (13) follows that $\dot{T} \leq f(T)$, hence

$$
\limsup _{t \rightarrow \infty} T(t) \leq T_{0},
$$

provided solutions to both systems are defined for all $t \geq 0$. To see that this is indeed the case, we argue by contradiction and let $\left(T(t), T^{*}(t), V(t)\right)$ be a solution with bounded maximal interval of existence $\mathcal{I}_{+}:=\left[0, t_{\max }\right)$. Then necessarily $T(t) \leq \max \left(T(0), T_{0}\right):=T_{\max }$ for all $t \in \mathcal{I}_{+}$. This implies that on $\mathcal{I}_{+}$, the following differential inequality holds for the solution of system (10) - (12):

$$
\begin{aligned}
\dot{T}^{*} & \leq P(\mu) K V T_{\max }-B T^{*} \\
\dot{V} & \leq \hat{N} B T^{*}-\Gamma V,
\end{aligned}
$$

or for system (13) - (15)

$$
\begin{aligned}
\dot{T}^{*} & \leq K V T_{\max }-B T^{*} \\
\dot{V} & \leq P(\mu) \hat{N} B T^{*}-\Gamma V,
\end{aligned}
$$

respectively. Notice that the right hand sides in the above inequalities are cooperative and linear vector fields. By a comparison principle for such inequalities we obtain that $T(t) \leq \tilde{T}(t)$ and $V(t) \leq \tilde{V}(t)$ (interpreted componentwise) for all $t$ in the intersection of the domains where the solutions are defined. Here, $(\tilde{T}(t), \tilde{V}(t))$ is the solution to the linear system whose vector field appears in the right hand side of the above inequalities, hence these solutions are defined for all $t \geq 0$. But then $T(t)$ and $V(t)$ can be extended continuously to the closed interval $\left[0, T_{\max }\right]$, contradicting maximality of $\mathcal{I}_{+}$.

Inequality (16) implies that for an arbitrary small $\epsilon>0$, there holds that $T(t) \leq T_{0}+\epsilon$ for all sufficiently large $t$. Now consider the behavior of the quantity $T+1^{\prime} T^{*}$ along solutions of both system (10) - (12) and (13) - (15):

$$
\frac{d}{d t}\left(T+1^{\prime} T^{*}\right)=f(T)-1^{\prime} B T^{*} \leq f(T)-b 1^{\prime} T^{*},
$$

where $b:=\min _{i}\left(\beta_{i}\right)$. By continuity of $f$ on the compact interval $\left[0, T_{0}+\epsilon\right]$, there exists (sufficiently large) $a>0$ such that

$$
f(T)+b T \leq a, \quad \text { for all } T \in\left[0, T_{0}+\epsilon\right] .
$$


Therefore, for all sufficiently large $t$, there holds that

$$
\frac{d}{d t}\left(T+1^{\prime} T^{*}\right) \leq a-b T-b 1^{\prime} T^{*} \leq a-b\left(T+1^{\prime} T^{*}\right),
$$

and hence

$$
\limsup _{t \rightarrow \infty} T(t)+1^{\prime} T^{*}(t) \leq \frac{a}{b} .
$$

Finally, from (12) and (15) follows that

$$
\limsup _{t \rightarrow \infty} V(t) \leq \frac{a}{b} \Gamma^{-1} \hat{N} B,
$$

and

$$
\limsup _{t \rightarrow \infty} V(t) \leq \frac{a}{b} \Gamma^{-1} P(\mu) \hat{N} B,
$$

respectively, where the limsup of a vector function is to be understood componentwise. Dissipativity now follows by observing that all the above bounds are independent of the initial condition.

Lemma 2. For $\mu=0$, let all single strain equilibria $E_{1}, E_{2} \ldots, E_{n}$ exist for either (10) - (12) or (13) - (15), and assume that

$$
\bar{T}^{1}<\bar{T}^{2}<\cdots<\bar{T}^{n}<\bar{T}^{n+1}:=T_{0},
$$

and

$$
f^{\prime}\left(\bar{T}^{j}\right) \leq 0, \text { for all } j=1, \ldots, n+1 .
$$

Then the Jacobian matrices of (10) - (12) or (13) - (15), evaluated at any of the $E_{i}$ 's, $i=1, \ldots, n+1$ (where $E_{n+1}:=E_{0}$ ) have the following properties: $J\left(E_{i}\right)$ has $i-1$ eigenvalues (counting multiplicities) in the open right half plane and $2(n+1)-i$ eigenvalues in the open left half plane. In particular, $J\left(E_{1}\right)$ is Hurwitz.

Proof. Note that when $\mu=0$, the Jacobian matrix associated to both model (10) - (12) and (13) - (15) is the same and given by:

$$
J=\left(\begin{array}{ccc}
f^{\prime}(T)-k^{\prime} V & 0 & -k^{\prime} T \\
K V & -B & K T \\
0 & \hat{N} B & -\Gamma
\end{array}\right) .
$$

To evaluate the Jacobian at any of the $E_{i}$ 's it is more convenient to reorder the components of the state vector by means of the following permutations:

1. For $i=1, \ldots, n$ we use $\left(T, T^{*}, V\right) \rightarrow\left(T, T_{i}^{*}, V_{i}, T_{1}^{*}, V_{1}, \ldots, T_{i-1}^{*}, V_{i-1}, T_{i+1}^{*}, V_{i+1}, \ldots, T_{n}^{*}, V_{n}\right)$.

2. For $i=n+1$ we use $\left(T, T^{*}, V\right) \rightarrow\left(T, T_{1}^{*}, V_{1}, T_{2}^{*}, V_{2}, \ldots, T_{n}^{*}, V_{n}\right)$.

Then the Jacobian matrices have the following structure:

1. For $i=1, \ldots, n$,

$$
J\left(E_{i}\right)=\left(\begin{array}{ccccccc}
A_{1}^{i} & * & \ldots & * & * & \ldots & * \\
0 & B_{1}^{i} & \ldots & 0 & 0 & \ldots & 0 \\
\vdots & \vdots & \ddots & \vdots & \vdots & \ldots & 0 \\
0 & 0 & \ldots & B_{i-1}^{i} & 0 & \ldots & 0 \\
0 & 0 & \ldots & 0 & B_{i+1}^{i} & \ldots & 0 \\
\vdots & \vdots & \ldots & \vdots & \vdots & \ddots & 0 \\
0 & 0 & \ldots & 0 & 0 & \ldots & B_{n}^{i}
\end{array}\right)
$$

where

$$
A_{1}^{i}=\left(\begin{array}{ccc}
f^{\prime}\left(\bar{T}^{i}\right)-k_{i} \bar{V}_{i} & 0 & -k_{i} \bar{T}^{i} \\
k_{i} \bar{V}_{i} & -\beta_{i} & k_{i} \bar{T}^{i} \\
0 & N_{i} \beta_{i} & -\gamma_{i}
\end{array}\right) \text { and } B_{l}^{i}=\left(\begin{array}{cc}
-\beta_{l} & k_{l} \bar{T}^{i} \\
N_{l} \beta_{l} & -\gamma_{l}
\end{array}\right), l \neq i,
$$

and therefore the eigenvalues of $J\left(E_{i}\right)$ coincide with those of $A_{1}^{i}$ and $B_{l}^{i}, l \neq i$. Since $f^{\prime}\left(\bar{T}^{i}\right) \leq 0$ it follows from lemma 3.4 in 1 that the eigenvalues of $A_{1}^{i}$ are in the open left half plane. The matrices $B_{l}^{i}$ are quasi-positive, irreducible matrices, hence by the Perron-Frobenius Theorem they have a simple real eigenvalue $\lambda_{l}^{i}$ with corresponding (componentwise) positive eigenvector. 
Notice that

$$
\operatorname{tr}\left(B_{l}^{i}\right)<0, \text { and } \operatorname{det}\left(B_{l}^{i}\right)=\beta_{l} \gamma_{l}\left(1-\frac{\bar{T}^{i}}{\bar{T}^{l}}\right)
$$

and thus by (21) that

$$
\lambda_{l}^{i}\left\{\begin{array}{l}
<0, \text { for all } l>i, \\
>0, \text { for all } l<i .
\end{array}\right.
$$

There are $i-1$ unstable $B$-blocks on the diagonal of $J\left(E_{i}\right)$, each of which contributes one positive eigenvalue to $J\left(E_{i}\right)$.

2. For $i=n+1$,

$$
J\left(E_{n+1}\right)=\left(\begin{array}{cccc}
A_{1}^{n+1} & * & \ldots & * \\
0 & B_{1}^{n+1} & \ldots & 0 \\
\vdots & \vdots & \ddots & \vdots \\
0 & 0 & \ldots & B_{n}^{n+1}
\end{array}\right)
$$

where

$$
A_{1}^{n+1}=\left(f^{\prime}\left(\bar{T}^{n+1}\right)\right) \text { and } B_{l}^{n+1}=\left(\begin{array}{cc}
-\beta_{l} & k_{l} \bar{T}^{n+1} \\
N_{l} \beta_{l} & -\gamma_{l}
\end{array}\right), l=1, \ldots, n .
$$

Notice that by a similar argument as in the previous case, all $n$ B-blocks on the diagonal of $J\left(E_{n+1}\right)$ are unstable with one positive and one negative eigenvalue.

When $\mu \neq 0$, the question arises as to what happens to the equilibria $E_{1}, \ldots, E_{n+1}$. The previous Lemma allows us to apply the Implicit Function Theorem which for small positive $\mu$ establishes the existence of (unique) equilibria $E_{j}(\mu)$ near each $E_{j}$. Indeed, denoting the vector field of either (10) - (12) or (13) - (15) by $F(X, \mu)$, we have that for all $j=1, \ldots, n+1$, there holds that $F\left(E_{j}, 0\right)=0$, and under the conditions of the previous Lemma we also have that $\partial F / \partial X\left(E_{j}, 0\right)$ is invertible. It is clear that $E_{n+1}(\mu)=E_{n+1}(0)$ for all $\mu \geq 0$, i.e. the disease-free equilibrium is not affected by mutations.

The main issue is of course whether or not the remaining equilibria $E_{j}(\mu), j=1, \ldots, n$ are non-negative. We study this problem next and derive results in terms of the properties of the mutation matrix $Q$.

For the steady-state analysis, we will need the following Lemma which is a relevant modification of Theorem A.12 (ii) in [13].

Lemma 3. Let $M$ be an irreducible square matrix with non-negative off-diagonal entries and let $s(M)$ be the stability modulus of $M$. Suppose that there exist $x, r \geq 0$ such that $M x+r=0$. Then the following hold:

1. If $s(M)>0$, then $x=r=0$;

2. If $s(M)=0$, then $r=0$ and $x$ is a multiple of the positive eigenvector of $M$.

Proof. Due to Perron-Frobenius Theorem, $s(M)$ is the principal eigenvalue of $M$. It is also the principal eigenvalue of $M^{\prime}$. Since $M^{\prime}$ is also irreducible and non-negative off-diagonal, there exists $v>0$ such that $M^{\prime} v=s(M) v$. Equivalently, $v^{\prime} M=s(M) v^{\prime}$. Hence

$$
0=v^{\prime}(M x+r)=s(M) v^{\prime} x+v^{\prime} r .
$$

If $s(M)>0$, then both non-negative products $v^{\prime} x$ and $v^{\prime} r$ must be zero which implies $x=r=0$. If $s(M)=0$, then $v^{\prime} r=0$ which implies $r=0$. Hence $M x=0=s(M) x$ so that $x$ is a multiple of the positive eigenvector of $M$.

For convenience, we introduce the following notation. We define $A(\mu):=\Gamma^{-1} \hat{N} P(\mu) K$ and assume (by renumbering the strains if necessary) that the strains are numbered in such a way that the matrix $A(\mu)$ has the lower block-triangular form

$$
A(\mu)=\left(\begin{array}{cccc}
A_{1}(\mu) & 0 & \ldots & 0 \\
\mu B_{2,1} & A_{2}(\mu) & \ldots & 0 \\
\vdots & \vdots & \ddots & \vdots \\
\mu B_{k, 1} & \mu B_{k, 2} & \ldots & \mu A_{k}(\mu)
\end{array}\right)
$$


where each diagonal block

$$
A_{i}(\mu)=\operatorname{diag}\left(\frac{1}{\bar{T}_{i 1}}, \ldots, \frac{1}{\bar{T}_{i s_{i}}}\right)+\mu B_{i}
$$

is such that $B_{i}, i=1, \ldots, k$ are irreducible with non-negative off-diagonal entries. The off-diagonal blocks $B_{i, j}, i>j$ are non-negative. We note that the diagonal entries of $A(0)$ are a permutation of

$$
0<\frac{1}{\bar{T}_{n}}<\frac{1}{\bar{T}_{n-1}}<\cdots<\frac{1}{\bar{T}_{1}}
$$

We say that the strain group $j$ is reachable from strain group $i$ if there exists a sequence of indices $i=l_{1}<$ $l_{2}<\ldots<l_{m}=j$ such that all matrices $B_{l_{s+1}, l_{s}}$ are nonzero. Our first result is as follows:

Proposition 1. Let the assumptions of Lemma国 hold, then the following hold:

1. For all sufficiently small $\mu>0$, matrix $A(\mu)$ admits $n$ distinct positive eigenvalues given by

$$
\frac{1}{\hat{T}_{n}(\mu)}<\frac{1}{\hat{T}_{n-1}(\mu)}<\cdots<\frac{1}{\hat{T}_{1}(\mu)},
$$

such that $\hat{T}_{i}(0)=\bar{T}_{i}$ for $i=1, \ldots, n$;

2. Matrix $A(\mu), \mu>0$ admits a positive eigenvector $\left(v_{1}, v_{2}, \ldots, v_{k}\right)$ if and only if $\frac{1}{\hat{T}_{1}(\mu)}$ is a principal eigenvalue of $A_{1}(\mu)$, and all strain groups $j \geq 2$ are reachable from strain group 1 ;

3. Matrix $A(\mu), \mu>0$ admits a non-negative eigenvector $\left(v_{1}, v_{2}, \ldots, v_{k}\right)$ for each eigenvalue $\frac{1}{T_{r}(\mu)}$ such that $\frac{1}{\hat{T}_{r}(\mu)}$ is a principal eigenvalue of some diagonal block $A_{i}(\mu)$, and $s\left(A_{j}(\mu)\right)<\frac{1}{\hat{T}_{r}(\mu)}$ for all $j=i+1, \ldots, k$ such that strain group $j$ is reachable from strain group $i$. The component $v_{j}$ is positive (zero) if group $j$ is reachable (not reachable) from strain group $i$.

4. All other eigenvectors of $A(\mu), \mu>0$ are not sign definite.

Proof. The first assertion follows readily because $A(0)$ has $n$ real distinct eigenvalues and $A(\mu)$ is continuous (actually, linear) in $\mu$. The continuity of eigenvalues with respect to $\mu$ implies that $\hat{T}_{i}(0)=\bar{T}_{i}$ for $i=1, \ldots, n$.

To prove the second assertion, we begin with sufficiency of the condition. Let $\mu>0$ be small and suppose that $\frac{1}{T_{1}(\mu)}$ is a principal eigenvalue of $A_{1}(\mu)$, and all strain groups $j \geq 2$ are reachable from strain group 1. Since $A_{1}(\mu)$ is irreducible with non-negative off-diagonal entries, Perron-Frobenius Theorem implies that the eigenvector $v_{1}$ associated with $\frac{1}{\hat{T}_{1}(\mu)}$ is positive. Since $\frac{1}{\hat{T}_{1}(\mu)}$ is also the principal eigenvalue of $A(\mu)$, it follows that

$$
s\left(A_{j}(\mu)-\frac{1}{\hat{T}_{1}(\mu)} I\right)<0, \quad j \geq 2,
$$

hence $\left(A_{j}(\mu)-\frac{1}{\hat{T}_{1}(\mu)} I\right)^{-1}<0$ (see e.g. Theorem A.12 (i) in [13]). The remaining components $v_{2}, \ldots, v_{k}$ of the eigenvector satisfy the triangular system

$$
\begin{aligned}
0 & =\mu B_{2,1} v_{1}+\left(A_{2}(\mu)-\frac{1}{T_{1}(\mu)} I\right) v_{2}, \\
0 & =\mu B_{3,1} v_{1}+\mu B_{3,2} v_{2}+\left(A_{3}(\mu)-\frac{1}{\hat{T}_{1}(\mu)} I\right) v_{3}, \\
\vdots & \vdots \\
0 & =\mu B_{k, 1} v_{1}+\cdots+\mu B_{k, k-1} v_{k-1}+\left(A_{k}(\mu)-\frac{1}{\hat{T}_{1}(\mu)} I\right) v_{k},
\end{aligned}
$$

Solving this system recursively, we obtain

$$
v_{j}=-\left(A_{j}(\mu)-\frac{1}{\hat{T}_{1}(\mu)} I\right)^{-1}\left(\mu B_{j, 1} v_{1}+\cdots+\mu B_{j, j-1} v_{j-1}\right), \quad j=2, \ldots, k .
$$

Since the strain group 2 is reachable from strain group 1 , the vector $\mu B_{2,1} v_{1} \geq 0$ is nonzero. Positivity of the matrix $-\left(A_{2}(\mu)-\frac{1}{\hat{T}_{1}(\mu)} I\right)^{-1}$ then implies that $v_{2}>0$. By induction on $j$, it follows that $v_{j}>0$ for all $j=2, \ldots, k$, and hence $v=\left(v_{1}, v_{2}, \ldots, v_{k}\right)$ is a positive eigenvector. 
To prove the converse (the necessary condition), let $v=\left(v_{1}, v_{2}, \ldots, v_{k}\right)$ be a positive eigenvector of $A(\mu)$ and let $\frac{1}{\hat{T}_{q}(\mu)}$ be the corresponding eigenvalue. Since $\left(A_{1}(\mu)-\frac{1}{\hat{T}_{q}(\mu)} I\right) v_{1}=0$ and $v_{1}>0, \frac{1}{\hat{T}_{q}(\mu)}$ must be the principal eigenvalue of $A_{1}(\mu)$ (Perron-Frobenius Thm). It remains to prove that $s\left(A_{j}(\mu)\right)<\frac{1}{T_{q}(\mu)}$ for all $j \geq 2$. Consider $j=2$, and for the sake of contradiction suppose that $s\left(A_{j}(\mu)-\frac{1}{\hat{T}_{q}(\mu)} I\right) \geq 0$. Since the eigenvalues are real and distinct for small $\mu>0$, this actually implies $s\left(A_{j}(\mu)-\frac{1}{\hat{T}_{q}(\mu)} I\right)>0$. Then we have that

$$
\left(A_{j}(\mu)-\frac{1}{\hat{T}_{q}(\mu)} I\right) v_{2}+\mu B_{2,1} v_{1}=0
$$

holds with non-negative vectors $v_{2}$ and $\mu B_{2,1} v_{1}$ which are both nonzero. By Lemma 3 , we have $v_{2}=0$, a contradiction. Hence $s\left(A_{2}(\mu)-\frac{1}{\hat{T}_{q}(\mu)} I\right)<0$. Proceeding by induction on $j$, we find that $s\left(A_{j}(\mu)-\frac{1}{\hat{T}_{q}(\mu)} I\right)<0$ for all $j \geq 2$. Therefore, $\frac{1}{\hat{T}_{q}(\mu)}$ must be the principal eigenvalue of $A(\mu)$, that is, $\frac{1}{\hat{T}_{q}(\mu)}=\frac{1}{\hat{T}_{1}(\mu)}$. This proves the second assertion.

To prove the third assertion, we again start with sufficient condition. Suppose that $\frac{1}{T_{r}(\mu)}$ is a principal eigenvalue of some diagonal block $A_{i}(\mu)$, and $s\left(A_{j}(\mu)\right)<\frac{1}{T_{r}(\mu)}$ for all $j=i+1, \ldots, k$ such that strain group $j$ is reachable from strain group $i$. It follows immediately that all matrices $A_{l}(\mu)-\frac{1}{\hat{T}_{r}(\mu)} I, l<i$ are nonsingular, and thus $v_{l}=0, l<i$. The component $v_{i}$ is the eigenvector of $A_{i}(\mu)-\frac{1}{T_{r}(\mu)} I$ and it is positive by Perron-Frobenius Theorem. Let $j=i+1$, then one of the following holds. If $i+1$ is not reachable from $i$, that is, $B_{i+1, i}=0$ so that

$$
\left(A_{i+1}(\mu)-\frac{1}{\hat{T}_{r}(\mu)} I\right) v_{i+1}=0
$$

implies $v_{i+1}=0$ because $A_{i+1}(\mu)-\frac{1}{\hat{T}_{r}(\mu)} I$ is nonsingular. If $i+1$ is reachable from $i$ and $s\left(A_{i+1}(\mu)-\frac{1}{\hat{T}_{r}(\mu)} I\right)<$ 0 , then

$$
v_{i+1}=-\left(A_{i+1}(\mu)-\frac{1}{\hat{T}_{r}(\mu)} I\right)^{-1} \mu B_{i+1, i} v_{i}>0 .
$$

By induction on $j$, it follows that $v_{j}=0$ for all $j>i$ that are not reachable from $i$ and $v_{j}>0$ for all $j>i$ that are reachable from $i$. Hence $v=\left(0, \ldots, 0, v_{i}, v_{i+1}, \ldots, v_{k}\right)$ is a non-negative eigenvector.

Now we prove the necessary condition of the third assertion. Let $v=\left(v_{1}, v_{2}, \ldots, v_{k}\right)$ be a non-negative eigenvector of $A(\mu)$ associated with eigenvalue $\frac{1}{\hat{T}_{r}(\mu)}$. Let $v_{i} \geq 0$ be the first nonzero component of $v$, that is, $v=\left(0, \ldots, 0, v_{i}, \ldots, v_{k}\right)$. Then $v_{i}$ satisfies $\left(A_{i}(\mu)-\frac{1}{T_{r}(\mu)} I\right) v_{i}=0$ hence $\frac{1}{\hat{T}_{r}(\mu)}$ must be an eigenvalue of $A_{i}(\mu)-\frac{1}{T_{r}(\mu)} I$. Moreover, by Perron-Frobenius Theorem, it must be the principal eigenvalue and $v_{i}>0$. Now consider $j=i+1$ and the equation

$$
\left(A_{i+1}(\mu)-\frac{1}{\hat{T}_{r}(\mu)} I\right) v_{i+1}+\mu B_{i+1, i} v_{i}=0 .
$$

The vectors $v_{i+1}$ and $\mu B_{i+1, i} v_{i}$ are non-negative. If $s\left(A_{i+1}(\mu)-\frac{1}{\hat{T}_{r}(\mu)} I\right)>0$ then by Lemma 3, $\mu B_{i+1, i} v_{i}=0$. Since $\mu>0$ and $v_{i}>0$, this implies $B_{i+1, i}=0$. Equivalently, $j=i+1$ is not reachable from $i$. An induction argument concludes the proof of the third assertion.

The final assertion of this Theorem is a simple one. Let $\frac{1}{\hat{T}_{r}(\mu)}$ be an eigenvalue of $A_{i}(\mu)$ but not the principal eigenvalue and let $v=\left(v_{1}, v_{2}, \ldots, v_{n}\right)$ be the corresponding eigenvector. Since all eigenvalues of $A(\mu)$ are distinct, the matrices $A_{l}(\mu)-\frac{1}{\hat{T}_{r}(\mu)} I, l<i$ are nonsingular so that $v_{l}=0, l<i$. Then $v_{i}$ must be an eigenvector of $A_{i}(\mu)$ and it cannot be sign definite due to Perron-Frobenius theorem. It follows that $v$ is not sign definite.

Our second result concerns the existence and the number of non-negative equilibria for the systems (10) - (12) and (13) - (15) with small $\mu>0$.

Proposition 2. Let the assumptions of Lemma 圆 hold and suppose that the strains are renumbered so that $A(\mu)$ has the form (23). Let $E_{j}(\mu)=\left(\hat{T}_{j}(\mu), \hat{T}_{j}^{*}(\mu), \hat{V}_{j}(\mu)\right)$ denote the nontrivial equilibria of both (10) - (12) and (13) - 15) for small $\mu>0$. Then

1. $E_{j}(\mu)$ is positive if and only if $\frac{1}{\hat{T}_{j}(\mu)}$ is an eigenvalue of $A(\mu)$ with a positive eigenvector $V_{j}$. 
2. $E_{j}(\mu)$ is non-negative if and only if $\frac{1}{\hat{T}_{j}(\mu)}$ is an eigenvalue of $A(\mu)$ with a non-negative eigenvector $V_{j}$.

3. $E_{j}(\mu) \notin \mathbb{R}_{+}^{2 n+1}$ if and only if $\frac{1}{\hat{T}_{j}(\mu)}$ is an eigenvalue of $A(\mu)$ with eigenvector $V_{j}$ which is not signdefinite.

Proof. We will prove the Proposition only for system (10) - (12) (the proof for (13) - (15) is similar). Observe that the equilibrium relation following from (12), can be expressed as $\hat{T}_{j}^{*}(\mu)=(\hat{N} B)^{-1} \Gamma \hat{V}_{j}(\mu)$. Hence, the signs of the corresponding components of $\hat{T}_{j}^{*}(\mu)$ and $\hat{V}_{j}(\mu)$ are the same. Substituting the above expression into (11) and (12), we find that $\hat{V}_{j}(\mu)$ must satisfy

$$
\left[\Gamma^{-1} \hat{N} P(\mu) K-\frac{1}{\hat{T}_{j}(\mu)} I\right] \hat{V}_{j}(\mu)=\left[A(\mu)-\frac{1}{\hat{T}_{j}(\mu)} I\right] \hat{V}_{j}(\mu)=0 .
$$

Thus for each nontrivial equilibrium $E_{j}(\mu)$, the quantity $\frac{1}{\hat{T}_{j}(\mu)}$ must be an eigenvalue of $A(\mu)$ and $\hat{V}_{j}(\mu)$ must be a multiple of the corresponding eigenvector $V_{j}$. If $V_{j}$ is not sign definite, it follows that $E_{j}(\mu) \notin \mathbb{R}_{+}^{2 n+1}$. For all $V_{j} \geq 0$, the components of $E_{j}(\mu)$ are uniquely determined via

$$
\hat{V}_{j}(\mu)=\frac{f\left(\hat{T}_{j}(\mu)\right)}{\hat{T}_{j}(\mu) k^{\prime} V_{j}} V_{j}, \quad \hat{T}_{j}^{*}(\mu)=(\hat{N} B)^{-1} \Gamma \hat{V}_{j}(\mu) .
$$

Hence $E_{j}(\mu)$ is positive (non-negative) if and only if $V_{j}$ is positive (non-negative).

An immediate corollary to Propositions 1 and 2 is that if the mutation matrix $Q$ is irreducible, then $A(\mu)$ is irreducible and systems (10) - (12) and (13) - (15) with small $\mu>0$ admit a unique positive equilibrium $E_{1}(\mu)$ and no other nontrivial non-negative equilibria. If the mutation matrix $Q$ is reducible, then positive equilibrium exists if and only the fittest strain (with lowest value $\bar{T}_{1}$ ) belongs to strain group 1 and all other strain groups are reachable from group 1, meaning that the fittest strain can eventually mutate into any other strain. In addition, nontrivial non-negative equilibria which are not positive are feasible for $\mu>0$ only if $Q$ is reducible. Specifically, if the strains can be numbered according to (23), then at most $k$ nontrivial non-negative equilibria exist. One extreme case is when the fittest strain belongs to group $k$, in which no positive and only one non-negative equilibrium exist. The opposite extreme case is $k=n$ where $A(\mu)$ is lower-triangular, the diagonal entries of $A(\mu)$ are arranged in decreasing order, and for any pair $i<j$, strain $j$ is reachable from strain $i$. In this case, there is a single positive equilibrium and $n-1$ non-negative equilibria.

\subsection{On uniform strong repellers}

Inspired by Thieme [15], we make the following definition.

Definition 1. Consider a system

$$
\dot{x}=F(x)
$$

on a compact forward invariant set $K \subset \mathbb{R}^{m}$ with a continuous flow $\phi(t, x)$. Let $K_{0} \subset K$ be a closed forward invariant subset of $K$. Let $d(x, A)$ denote the distance from a point $x$ to the set $A$. We say that $K_{0}$ is a uniform strong repeller in $K$ if there exists a $\delta>0$ such that for all solutions $\phi(t, x) \in K \backslash K_{0}$, $\liminf _{t \rightarrow \infty} d\left(\phi(t, x), K_{0}\right) \geq \delta$.

Theorem 3. Let $\Pi: K \rightarrow \mathbb{R}^{+}$be a continuously differentiable function such that $\Pi(x)=0$ if and only if $x \in K_{0}$. Suppose there exists a lower semi-continuous function $\psi: K \rightarrow \mathbb{R}$ such that

$$
\frac{\dot{\Pi}}{\Pi}=\psi, \quad \forall x \in K \backslash K_{0} .
$$

Suppose that the following condition holds

$$
\text { (H) } \forall x \in K_{0}, \exists T>0:\langle\psi(\phi(T, x))\rangle>0 .
$$

Then $K_{0}$ is a uniform strong repellor in $K$. 
Proof. Step 1. Note that by lower semi-continuity of $\psi$ and continuity of $\phi$, for every $p \in K_{0}$ we can find an open set $U_{p}$ containing $p$, and a lower semi-continuous map $T_{p}: U_{p} \rightarrow(0,+\infty)$ so that for every $q \in U_{p},(\mathrm{H})$ holds with $x=q$ and $T=T_{p}(q)$. Choose for every $p \in K_{0}$ a non-empty open set $V_{p}$ with $\bar{V}_{p} \subset U_{p}$. Then by lower semi-continuity of each map $T_{p}$ and compactness of $\bar{V}_{p}$,

$$
\inf _{q \in \bar{V}_{p}} T_{p}(q)>0
$$

is achieved in $\bar{V}_{p}$. Since $\cup_{p \in K_{0}} V_{p}$ is an open cover of $K_{0}$, we may choose a finite open subcover $\cup_{i=1, \ldots, n} V_{p_{i}}$. Let $\tau_{i}=\inf _{q \in \bar{V}_{p_{i}}} T_{p_{i}}(q)>0$ and set

$$
\tau=\min _{i=1, \ldots, n} \tau_{i}>0 .
$$

Note that for every $p \in K_{0}$, there is a $T \geq \tau$ so that $(\mathrm{H})$ holds with $x=p$. That is, $\tau$ is a uniform (in $K_{0}$ ) lower bound for $T$ 's for which $(\mathrm{H})$ holds.

Step 2. Let $h>0$ be given. Define

$$
U_{h}=\{x \in K \mid \exists T>\tau:\langle\psi(\phi(T, x))\rangle>h\}
$$

We claim that $U_{h}$ is open.

Fix $z \in U_{h}$. Then there is some $T>\tau$ so that

$$
\epsilon:=\langle\psi(\phi(T, z))\rangle-h>0 .
$$

Then by continuity of $\phi$ and lower semi-continuity of $\psi$ (and therefore uniform lower semi-continuity of $\psi$ on compact sets), it follows that there exists an open set $W_{z}$ containing $z$ such that for all $z^{\prime} \in W_{z}$ holds that

$$
\psi\left(\phi\left(t, z^{\prime}\right)>\psi(\phi(t, z))-\epsilon, \quad \forall t \in[0, T] .\right.
$$

Now since

$$
\langle\psi(\phi(T, z))\rangle=\epsilon+h
$$

it follows from (27) that for all $z^{\prime} \in W_{z}$ :

$$
\left\langle\psi\left(\phi\left(T, z^{\prime}\right)\right)\right\rangle>h
$$

and thus that $W_{z} \subset U_{h}$, establishing our claim.

Step 3. Define $T_{h}: U_{h} \rightarrow[\tau,+\infty)$ as

$$
T_{h}(z)=\inf \{T>\tau \mid\langle\psi(\phi(T, z))\rangle>h\} .
$$

We claim that $T_{h}$ is upper semi-continuous.

Fix $z \in U_{h}$ and let $\epsilon^{\prime}>0$ be given. Then there is some $T>\tau$ so that

$$
\langle\psi(\phi(T, z))\rangle>h,
$$

so that

$$
T<T_{h}(z)+\epsilon^{\prime}
$$

By the argument in Step 2, there is some open set $W_{z}$ containing $z$, such that for all $z^{\prime} \in W_{z}$ holds that:

$$
\left\langle\psi\left(\phi\left(T, z^{\prime}\right)\right)\right\rangle>h,
$$

and thus that for all $z^{\prime} \in W_{z}$ :

$$
T_{h}\left(z^{\prime}\right) \leq T
$$

Our claim follows by combining (28) and (29).

Step 4. The nested family $\left\{U_{h}\right\}_{h>0}$ is decreasing (under set inclusion), and forms an open cover of $K_{0}$. Hence, there is some $\bar{h}$ so that $U_{\bar{h}}$ covers $K_{0}$. Since $\tilde{K}:=K \backslash U_{\bar{h}}$ is compact, and $\Pi$ is continuous, $\Pi$ attains its minimal value $m>0$ on $\tilde{K}$. Choose $p \in(0, m)$ and define:

$$
I_{p}=\{z \in K \mid \Pi(z) \in(0, p]\} .
$$

Then $I_{p} \subset U_{\bar{h}}$. 
Step 5. We claim that every forward solution starting in $I_{p}$, eventually leaves $I_{p}$, that is:

$$
\forall z \in I_{p}, \exists t_{z}>0: \quad \phi\left(t_{z}, z\right) \notin I_{p} .
$$

By contradiction, if $\phi(t, z) \in I_{p}$ for all $t \geq 0$, then $\phi(t, z) \in U_{\bar{h}}$ for all $t \geq 0$, and thus:

$$
\exists T_{t} \geq \tau: \frac{1}{T_{t}} \int_{t}^{t+T_{t}} \psi(\phi(s, z)) d s>\bar{h} .
$$

Then integrating equation (25) from $t$ to $t+T$ yields that:

$$
\ln \left(\frac{\Pi\left(\phi\left(t+T_{t}, z\right)\right)}{\Pi(\phi(t, z))}\right)>\bar{h} T_{t},
$$

and thus that

$$
\Pi\left(\phi\left(t+T_{t}, z\right)\right)>e^{\bar{h} T_{t}} \Pi(\phi(t, z)) .
$$

Set $t_{0}=0$ and $t_{k}=t_{k-1}+T_{t_{k-1}}$ for $k=1,2, \ldots$ Since each $T_{t_{k}} \geq \tau>0$ it follows that $t_{k} \rightarrow \infty$. Then by (30) and since $t_{k} \geq \tau$ for all $k$, we have that:

$$
\Pi\left(\phi\left(t_{k}, z\right)\right)>e^{\bar{h} T_{t_{k-1}}} \Pi\left(\phi\left(t_{k-1}, z\right)\right)>e^{k \tau} \Pi(z),
$$

so that $\Pi\left(\phi\left(t_{k}, z\right)\right) \rightarrow \infty$ as $k \rightarrow \infty$. This contradicts boundedness of $\Pi$ on the compact set $K$.

Step 6. Let

$$
\tilde{I}_{p}=I_{p} \cup K_{0}
$$

We will show that there is some $q \in(0, p)$ so that forward solutions starting outside $\tilde{I}_{p}$, never reach $I_{q}$, that is:

$$
\exists q \in(0, p): \quad z \notin \tilde{I}_{p} \Rightarrow \phi(t, z) \notin I_{q}, \quad \forall t \geq 0 .
$$

Consider a forward solution $\phi(t, z)$ with $z \notin \tilde{I}_{p}$. If $\phi(t, z) \notin \tilde{I}_{p}$ for all $t \geq 0$, then we are done since $\tilde{I}_{q} \subset \tilde{I}_{p}$, so let us assume that for some $t_{z}>0$, holds that $\phi\left(t_{z}, z\right) \in \tilde{I}_{p}$. Denote the first time this happens by $t_{0}$ :

$$
t_{0}=\min \left\{t>0 \mid \phi(t, z) \in \tilde{I}_{p}\right\} .
$$

Set $z^{*}=\phi\left(t_{0}, z\right)$ and note that $\Pi\left(z^{*}\right)=p$. Denote $\inf _{z \in K_{0}} \psi(z)$ by $m^{\prime}$. If $m^{\prime} \geq 0$, then (25) implies that $\Pi\left(\phi\left(t, z^{*}\right)\right) \geq \Pi\left(z^{*}\right)=p$ for all $t \geq 0$, so that we're done. If on the the other hand $m^{\prime}<0$, we first define

$$
\bar{T}=\max _{z \in \tilde{I}_{p}} T_{h}(z)(\geq \tau>0)
$$

Notice that this maximum is indeed achieved on the compact set $\tilde{I}_{p}$, since $T_{h}$ is upper semi-continuous. Now we define

$$
q=p e^{m^{\prime} \bar{T}}
$$

and notice that $q$ is independent of the chosen solution $z(t)$. We will show that for this choice of $q$, our claim is established.

We have that:

$$
\forall t \in(0, \bar{T}): \frac{1}{t} \int_{0}^{t} \psi\left(\phi\left(s, z^{*}\right)\right) d s \geq m^{\prime}
$$

and thus by (25) that

$$
\forall t \in(0, \bar{T}): \Pi\left(\phi\left(t, z^{*}\right)\right) \geq \Pi\left(z^{*}\right) e^{m^{\prime} t}>q,
$$

which implies that during the time interval $(0, \bar{T})$, the solution $\phi\left(t, z^{*}\right)$ has not reached $I_{q}$. On the other hand, during that same time interval $(0, \bar{T})$, the solution $\phi\left(t, z^{*}\right)$ must have left $\tilde{I}_{p}$. If this were not the case, then by the argument in Step 5 , there would be some $T^{*} \in[\tau, \bar{T})$ so that

$$
\Pi\left(\phi\left(T^{*}, z^{*}\right)\right) \geq \Pi\left(z^{*}\right) e^{\bar{h} T}>p
$$

and thus that $\phi\left(T^{*}, z^{*}\right) \notin \tilde{I}_{p}$, a contradiction to our assumption. This process can be repeated iteratively and leads to the conclusion that the forward solution $\phi(t, z)$ which did not start in $\tilde{I}_{p}$, will never reach $I_{q}$.

So far we have shown that for any solution $\phi(t, x) \notin K_{0}$, inequality $\Pi(\phi(t, x)) \geq q>0$ for all sufficiently large $t$. The sets $K_{0}=\Pi^{-1}(\{0\})$ and $\Pi^{-1}([q,+\infty)) \cap K$ are compact and disjoint. Therefore, there exists $\delta>0$ such that $d\left(\phi(t, x), K_{0}\right) \geq \delta$ for all $x \notin K_{0}$ and all sufficiently large $t$. 


\subsection{Global stability for small $\mu>0$}

The following Lemmas will be used to prove global stability of the positive equilibrium for small $\mu>0$.

Lemma 4. Let $a: \mathbb{R}^{m} \rightarrow \mathbb{R}^{n}$ be continuous and let $b \in \operatorname{int}\left(\mathbb{R}_{+}^{n}\right)$. Let $f: \mathbb{R}^{m} \times\left(\mathbb{R}_{+}^{n} \backslash\{0\}\right) \rightarrow \mathbb{R}$ be defined as

$$
f(x, y)=\frac{a^{\prime}(x) y}{b^{\prime} y}
$$

Then

$$
\liminf _{x \rightarrow x_{0}, y \rightarrow 0+} f(x, y)=\min _{i \in\{1, \ldots, n\}} \frac{a_{i}\left(x_{0}\right)}{b_{i}},
$$

furthermore, if we define $f(x, 0)=\min _{i \in\{1, \ldots, n\}} \frac{a_{i}(x)}{b_{i}}$, then $f(x, y)$ becomes a lower semi-continuous function on $\mathbb{R}^{m} \times \mathbb{R}_{+}^{n}$ whose restriction on $\mathbb{R}^{m} \times\{0\}$ is continuous.

Proof. Extending the function $f(x, y)$ by defining $f\left(x_{0}, 0\right)=\liminf _{x \rightarrow x_{0}, y \rightarrow 0+} f(x, y)$ clearly produces a lower semi-continuous function. Furthermore, since $a(x)$ is continuous, the function $\min _{i \in\{1, \ldots, n\}} \frac{a_{i}(x)}{b_{i}}$ is continuous as well. So it remains to show that (32) holds.

Without loss of generality, we may assume that $\min _{i \in\{1, \ldots, n\}} \frac{a_{i}\left(x_{0}\right)}{b_{i}}=\frac{a_{1}\left(x_{0}\right)}{b_{1}}$. Setting $x=x_{0}$ and $y_{2}=$ $y_{3}=\ldots=y_{n}=0$ and letting $y_{1} \rightarrow 0^{+}$, we find that $f\left(x_{0}, y_{1}, 0, \ldots, 0\right) \rightarrow \frac{a_{1}\left(x_{0}\right)}{b_{1}}$. Hence, $\liminf _{x \rightarrow x_{0}, y \rightarrow 0+} f(x, y) \leq$ $\frac{a_{1}\left(x_{0}\right)}{b_{1}}$. We also observe that as long as $y \in \mathbb{R}_{+}^{n} \backslash\{0\}$, the value

$$
\frac{a^{\prime}(x) y}{b^{\prime} y}=\sum_{i=1}^{n} \frac{a_{i}(x)}{b_{i}} \frac{b_{i} y_{i}}{b_{1} y_{1}+\cdots+b_{n} y_{n}}
$$

is a convex linear combination of the values $\frac{a_{i}(x)}{b_{i}}, i=1, \ldots, n$. By continuity of $a(x)$, for any $\varepsilon>0$ there exists $\delta>0$ such that $\forall i \in\{1, \ldots, n\}$ and $\forall x \in B_{\delta}\left(x_{0}\right)$, we have $a_{i}(x)>a_{i}\left(x_{0}\right)-\varepsilon b_{i}$. Hence, for all $x \in B_{\delta}\left(x_{0}\right)$ and for all $y \in \mathbb{R}_{+}^{n} \backslash\{0\}, f(x, y) \geq \frac{a_{1}\left(x_{0}\right)}{b_{1}}-\varepsilon$. We have established that

$$
\frac{a_{1}\left(x_{0}\right)}{b_{1}} \geq \liminf _{x \rightarrow x_{0}, y \rightarrow 0+} f(x, y) \geq \frac{a_{1}\left(x_{0}\right)}{b_{1}}-\varepsilon .
$$

Since $\varepsilon>0$ is arbitrary, (32) follows.

Lemma 5. Suppose that (8) holds. Then there exist $\eta, \mu_{0}>0$ such that

$$
\liminf _{t \rightarrow \infty} 1^{\prime} V(t) \geq \eta>0
$$

for any $\mu \in\left[0, \mu_{0}\right]$ and for any solution of (10) - (12) and (13) - (15) with $1^{\prime} V(t)>0$.

Proof. We will prove the claim for system (10) - (12) (the proof for (13) - (15) is similar). The proof consists of two parts. We first show that there exist $\eta_{0}, \mu_{0}>0$ such that $\liminf _{t \rightarrow \infty} 1^{\prime}\left(T^{*}(t)+V(t)\right) \geq \eta_{0}>0$ for all solutions with $T^{*}(t), V(t) \neq 0$.

We choose $n$ positive numbers $\tilde{N}_{i}$ so that $\frac{\gamma_{i}}{k_{i} T_{0}}<\tilde{N}_{i}<N_{i}$ for all $1 \leq i \leq n$. This is possible since we assume $\bar{T}_{i}=\frac{\gamma_{i}}{k_{i} N_{i}}<T_{0}$. Let $v=(\tilde{N}, 1)$. It follows that

$$
v^{\prime}\left(\begin{array}{cc}
-B & K T_{0} \\
\hat{N} B & -\Gamma
\end{array}\right)=\left(b_{1}\left(N_{1}-\tilde{N}_{1}\right), \ldots, b_{n}\left(N_{n}-\tilde{N}_{n}\right), k_{1} T_{0} \tilde{N}_{1}-\gamma_{1}, \ldots, k_{n} T_{0} \tilde{N}_{n}-\gamma_{n}\right)
$$

is a positive vector. By continuity, there exists a $\mu_{0}>0$ such that

$$
v^{\prime} M(T, \mu), \quad \text { where } M(T, \mu):=\left(\begin{array}{cc}
-B & P(\mu) K T \\
\hat{N} B & -\Gamma
\end{array}\right)
$$

is a positive vector for all $\mu \in\left[0, \mu_{0}\right]$.

Consider a system

$$
\begin{aligned}
\dot{T} & =f(T)-k^{\prime} V T, T \in \mathbb{R}_{+} \\
\dot{T}^{*} & =P(\mu) K V T-B T^{*}, T^{*} \in \mathbb{R}_{+}^{n}, \\
\dot{V} & =\hat{N} B T^{*}-\Gamma V, V \in \mathbb{R}_{+}^{n}, \\
\dot{\mu} & =0, \mu \in\left[0, \mu_{0}\right] .
\end{aligned}
$$


Let $K^{\prime}$ be the forward invariant compact set for (10)- (12) established in Lemma[1]and define $K=K^{\prime} \times\left[0, \mu_{0}\right]$. It is clear that $K$ is compact and forward invariant under (33) - (36) The set

$$
K_{0}:=\left(\left[0, T_{0}\right] \times\{0\} \times\{0\} \times\left[0, \mu_{0}\right]\right) \cap K
$$

is clearly a compact forward invariant subset of $K$.

Let $\Pi\left(T^{*}, V\right):=v^{\prime}\left(T^{*}, V\right)$. The function $\Pi$ is clearly smooth, zero on $K_{0}$, and positive on $K \backslash K_{0}$. Furthermore,

$$
\frac{\dot{\Pi}}{\Pi}=\psi:=\frac{v^{\prime} M(T, \mu)\left(T^{*}, V\right)}{v^{\prime}\left(T^{*}, V\right)}
$$

is lower semi-continuous on $K$ by Lemma 4 once we define the value of $\psi$ on $K_{0}$ as

$$
\psi(T, \mu)=\min _{i=1, \ldots, n} \frac{v^{\prime} M(T, \mu)_{i}}{v_{i}} .
$$

We note that the function $\psi(T, \mu)$ is continuous in $(T, \mu)$. Since all solutions of (33) $-(36)$ in $K_{0}$ have the property that $\lim _{t \rightarrow \infty} T(t)=T_{0}$, it implies that $\psi(T(t), \mu)>0$ for all sufficiently large $t$. Hence by Theorem 3, the set $K_{0}$ is a uniform strong repeller in $K$. If we use the $L^{1}$-norm of $\left(T^{*}, V\right)$ as the distance function to $K_{0}$, we find that there exists an $\eta_{0}>0$ such that

$$
\liminf _{t \rightarrow \infty} 1^{\prime}\left(T^{*}+V\right) \geq \eta_{0}
$$

for all solutions of $\left(\underline{33}-\underline{36}\right.$ in $K \backslash K_{0}$.

To complete the proof, we need to show that there exists $\eta>0$ such that $\liminf _{t \rightarrow \infty} 1^{\prime} V(t) \geq \eta>0$ for all solutions with $1^{\prime} V(t)>0$. Observe that $1^{\prime} V(t)>0$ implies that $1^{\prime} T^{*}(t)>0$. Hence by the result of part one, we have that $\liminf _{t \rightarrow \infty} 1^{\prime}\left(T^{*}(t)+V(t)\right) \geq \eta_{0}>0$, or equivalently, $1^{\prime} T^{*}(t)>\eta_{0} / 2-1^{\prime} V(t)$ for all sufficiently large $t$. We substitute this inequality into (12) and find that

$$
1^{\prime} \dot{V} \geq A_{0}\left(\frac{\eta_{0}}{2}-1^{\prime} V(t)\right)-A_{1} 1^{\prime} V(t), \quad A_{0}:=\min _{i}\left(N_{i} \beta_{i}\right)>0, A_{1}:=\max _{i}\left(\gamma_{i}\right)>0
$$

holds for large $t$. It follows immediately that

$$
\liminf _{t \rightarrow \infty} 1^{\prime} V(t) \geq \eta=\frac{\eta_{0} A_{0}}{2\left(A_{0}+A_{1}\right)}>0 .
$$

Lemma 6. Let

$$
\sigma(x, y, z):=x+y+\frac{z}{x y}-3 z^{\frac{1}{3}}
$$

Then for any $z_{0}, M>0$, there exists $\delta>0$ such that $\sigma(x, y, z)>M$ for all $0<x<\delta$, all $y>0$, and all $z>z_{0}$.

Proof. Observe that the minimum of the function $\sigma(x, \cdot, z)$ on the set $y \in(0,+\infty)$ is achieved at $y=\sqrt{z / x}$. Hence for all $y>0$, it holds that

$$
f(x, y, z) \geq f\left(x, \sqrt{\frac{z}{x}}, z\right)=x+2 \sqrt{\frac{z}{x}}-3 z^{\frac{1}{3}} .
$$

Let $z_{0}>0$ and define

$$
\delta:=\frac{4 z_{0}}{\left(M+3 z_{0}^{\frac{1}{3}}\right)^{2}} .
$$

Then for all $0<x<\delta$, all $y>0$, and all $z>z_{0}$, it holds that

$$
f(x, y, z) \geq 2 \sqrt{\frac{z}{x}}-3 z^{\frac{1}{3}}=z^{\frac{1}{2}}\left(\frac{2}{x^{\frac{1}{2}}}-3 z^{-\frac{1}{6}}\right)>z_{0}^{\frac{1}{2}}\left(\frac{2}{\delta^{\frac{1}{2}}}-3 z_{0}^{-\frac{1}{6}}\right)=M .
$$


Theorem 4. Let $K$ be the absorbing compact set established in Lemma 1 , and let

$$
U=\left\{\left(T, T^{*}, V\right) \in \mathbb{R}_{+}^{2 n+1} \mid T, T_{1}^{*}, V_{1}>0\right\} .
$$

Suppose that $(\mathbf{C})$ holds with $\bar{T}_{1}$ instead of $\bar{T}$. Then there exist $\mu_{1}>0$ and a compact set $K_{\delta} \subset U$ such that for any $\mu \in\left[0, \mu_{1}\right]$ and for any solution of (10) - (12) or (13) - (15) in $U$, there exists a $t_{0}>0$ such that $\left(T(t), T^{*}(t), V(t)\right) \in K_{\delta}$ for all $t>t_{0}$.

Proof. Both for system (10) - (12) and (13) - (15), the proof will be based on the same Lyapunov function

$$
W=\int_{\bar{T}^{1}}^{T}\left(1-\frac{\bar{T}^{1}}{\tau}\right) d \tau+\int_{\bar{T}_{1}^{*}}^{T_{1}^{*}}\left(1-\frac{\bar{T}_{1}^{*}}{\tau}\right) d \tau+\frac{1}{N_{1}} \int_{\bar{V}_{1}}^{V_{1}}\left(1-\frac{\bar{V}_{1}}{\tau}\right) d \tau+\sum_{i>1} T_{i}^{*}+\frac{1}{N_{i}} V_{i}
$$

that we used to show competitive exclusion with $\mu=0$.

Case 1: System (10) - (12).

Computing $\dot{W}$ for system (10) - (12), we obtain after some simplifications

$$
\begin{aligned}
\dot{W}= & \left(f(T)-f\left(\bar{T}^{1}\right)\right)\left(1-\frac{\bar{T}^{1}}{T}\right)-\beta_{1} \bar{T}_{1}^{*}\left[\frac{\bar{T}^{1}}{T}+\frac{\bar{T}_{1}^{*} V_{1} T}{T_{1}^{*} \bar{V}_{1} \bar{T}^{1}}+\frac{\bar{V}_{1} T_{1}^{*}}{V_{1} \bar{T}_{1}^{*}}-3\right] \\
& -\sum_{i=2}^{n} k_{i} V_{i}\left(\bar{T}^{i}-\bar{T}^{1}\right)+\frac{T_{1}^{*}-\bar{T}_{1}^{*}}{T_{1}^{*}} \mu \sum_{j=1}^{n} q_{1 j} k_{j} V_{j} T+\mu \sum_{i=2}^{n} \sum_{j=1}^{n} q_{i j} k_{j} V_{j} T .
\end{aligned}
$$

Recombining the terms, we further obtain

$$
\begin{aligned}
\dot{W}= & \left(f(T)-f\left(\bar{T}^{1}\right)\right)\left(1-\frac{\bar{T}^{1}}{T}\right)-\beta_{1} \bar{T}_{1}^{*}\left[\frac{\bar{T}^{1}}{T}+\frac{\bar{T}_{1}^{*} V_{1} T}{T_{1}^{*} \bar{V}_{1} \bar{T}^{1}}+\frac{\bar{V}_{1} T_{1}^{*}}{V_{1} \bar{T}_{1}^{*}}-3\right] \\
& -\sum_{i=2}^{n} k_{i} V_{i}\left(\bar{T}^{i}-\bar{T}^{1}\right)-\frac{\bar{T}_{1}^{*}}{T_{1}^{*}} \mu \sum_{j=1}^{n} q_{1 j} k_{j} V_{j} T+\mu \sum_{i=1}^{n} \sum_{j=1}^{n} q_{i j} k_{j} V_{j} T .
\end{aligned}
$$

We note that

$$
\sum_{i=1}^{n} \sum_{j=1}^{n} q_{i j} k_{j} V_{j} T=\sum_{j=1}^{n}\left(\sum_{i=1}^{n} q_{i j}\right) k_{j} V_{j} T=0
$$

since all column sums of $Q$ are zero. Hence,

$$
\begin{aligned}
\dot{W}= & \left(f(T)-f\left(\bar{T}^{1}\right)\right)\left(1-\frac{\bar{T}^{1}}{T}\right)-\beta_{1} \bar{T}_{1}^{*}\left[\frac{\bar{T}^{1}}{T}+\frac{\bar{T}_{1}^{*} V_{1} T}{T_{1}^{*} \bar{V}_{1} \bar{T}^{1}}+\frac{\bar{V}_{1} T_{1}^{*}}{V_{1} \bar{T}_{1}^{*}}-3\right] \\
& -\sum_{i=2}^{n} k_{i} V_{i}\left(\bar{T}^{i}-\bar{T}^{1}\right)-\frac{\bar{T}_{1}^{*}}{T_{1}^{*}} \mu q_{11} k_{1} V_{1} T-\frac{\bar{T}_{1}^{*}}{T_{1}^{*}} \mu \sum_{j=2}^{n} q_{1 j} k_{j} V_{j} T .
\end{aligned}
$$

We rewrite $\dot{W}$ as

$$
\begin{aligned}
\dot{W}= & \left(f(T)-f\left(\bar{T}^{1}\right)\right)\left(1-\frac{\bar{T}^{1}}{T}\right)-\beta_{1} \bar{T}_{1}^{*}\left[\frac{\bar{T}^{1}}{T}+\left(1+q_{11} \mu\right) \frac{\bar{T}_{1}^{*} V_{1} T}{T_{1}^{*} \bar{V}_{1} \bar{T}^{1}}+\frac{\bar{V}_{1} T_{1}^{*}}{V_{1} \bar{T}_{1}^{*}}-3\left(1+q_{11} \mu\right)^{1 / 3}\right] \\
& +3 \beta_{1} \bar{T}_{1}^{*}\left(1-\left(1+q_{11} \mu\right)^{1 / 3}\right)-\sum_{i=2}^{n} k_{i} V_{i}\left(\bar{T}^{i}-\bar{T}^{1}\right)-\frac{\bar{T}_{1}^{*}}{T_{1}^{*}} \mu \sum_{j=2}^{n} q_{1 j} k_{j} V_{j} T .
\end{aligned}
$$

Note that the last term of $\dot{W}$ is non-positive, henc $\varepsilon^{1}$

$$
\begin{aligned}
\dot{W} \leq & \left(f(T)-f\left(\bar{T}^{1}\right)\right)\left(1-\frac{\bar{T}^{1}}{T}\right)-\beta_{1} \bar{T}_{1}^{*}\left[\frac{\bar{T}^{1}}{T}+\left(1+q_{11} \mu\right) \frac{\bar{T}_{1}^{*} V_{1} T}{T_{1}^{*} \bar{V}_{1} \bar{T}^{1}}+\frac{\bar{V}_{1} T_{1}^{*}}{V_{1} \bar{T}_{1}^{*}}-3\left(1+q_{11} \mu\right)^{1 / 3}\right] \\
+ & 3 \beta_{1} \bar{T}_{1}^{*}\left(1-\left(1+q_{11} \mu\right)^{1 / 3}\right)-\sum_{i=2}^{n} k_{i} V_{i}\left(\bar{T}^{i}-\bar{T}^{1}\right) .
\end{aligned}
$$

\footnotetext{
${ }^{1}$ Incidentally, if $q_{11}=0$, we obtain global stability of the boundary equilibrium $E_{1}$ for all $\mu>0$.
} 
By Lemma 5. there exist $\eta, \mu_{a}>0$ such that $1^{\prime} V(t)>\eta$ for all $\mu \in\left[0, \mu_{a}\right]$ and all sufficiently large $t$. Let $\alpha=\min _{i \geq 2} k_{i}\left(\bar{T}^{i}-\bar{T}^{1}\right)>0$, then

$$
\sum_{i=2}^{n} k_{i} V_{i}\left(\bar{T}^{i}-\bar{T}^{1}\right) \geq \alpha \sum_{i=2}^{n} V_{i} \geq \alpha\left(\eta-V_{1}\right)
$$

Thus, by shifting time forward if necessary, we have the inequality

$$
\begin{aligned}
& \dot{W} \leq\left(f(T)-f\left(\bar{T}^{1}\right)\right)\left(1-\frac{\bar{T}^{1}}{T}\right)-\beta_{1} \bar{T}_{1}^{*}\left[\frac{\bar{T}^{1}}{T}+\left(1+q_{11} \mu\right) \frac{\bar{T}_{1}^{*} V_{1} T}{T_{1}^{*} \bar{V}_{1} \bar{T}^{1}}+\frac{\bar{V}_{1} T_{1}^{*}}{V_{1} \bar{T}_{1}^{*}}-3\left(1+q_{11} \mu\right)^{1 / 3}\right] \\
&+3 \beta_{1} \bar{T}_{1}^{*}\left(1-\left(1+q_{11} \mu\right)^{1 / 3}\right)-\alpha \eta+\alpha V_{1} .
\end{aligned}
$$

Let $\mu_{b}>0$ be such that for all $\mu \in\left[0, \mu_{b}\right]$,

$$
1+q_{11} \mu \in\left[\frac{1}{2}, 1\right], \quad 3 \beta_{1} \bar{T}_{1}^{*}\left(1-\left(1+q_{11} \mu\right)^{1 / 3}\right)-\alpha \eta \leq-\frac{\alpha \eta}{2} .
$$

Let $\mu_{1}=\min \left(\mu_{a}, \mu_{b}\right)$ and choose sufficiently large $L>0$ so that

$$
3 \beta_{1} \bar{T}_{1}^{*}\left(1-\left(1+q_{11} \mu\right)^{1 / 3}\right)-\alpha \eta+\alpha V_{1}<L
$$

for all solutions of (10) - (12) in $K$ and all $\mu \in\left[0, \mu_{1}\right]$. For any $\mu \in\left[0, \mu_{1}\right]$, we have that

$$
\begin{aligned}
\dot{W} \leq & \left(f(T)-f\left(\bar{T}^{1}\right)\right)\left(1-\frac{\bar{T}^{1}}{T}\right)-\beta_{1} \bar{T}_{1}^{*}\left[\frac{\bar{T}^{1}}{T}+\left(1+q_{11} \mu\right) \frac{\bar{T}_{1}^{*} V_{1} T}{T_{1}^{*} \bar{V}_{1} \bar{T}^{1}}+\frac{\bar{V}_{1} T_{1}^{*}}{V_{1} \bar{T}_{1}^{*}}-3\left(1+q_{11} \mu\right)^{1 / 3}\right] \\
& -\frac{\alpha \eta}{2}+\alpha V_{1},
\end{aligned}
$$

where the first two terms are non-positive and $1+q_{11} \mu \in\left[\frac{1}{2}, 1\right]$. Inspecting the first term in $\dot{W}$, we find that there exists $\delta_{0}>0$ such that

$$
\left(f(T)-f\left(\bar{T}^{1}\right)\left(1-\frac{\bar{T}^{1}}{T}\right)<-(L+1)\right.
$$

for all $T<\delta_{0}$ and all $\mu \in\left[0, \mu_{1}\right]$. Now we inspect the the second term in $\dot{W}$. Using Lemma 6 with

$$
x=\frac{\bar{V}_{1} T_{1}^{*}}{V_{1} \bar{T}_{1}^{*}}, \quad y=\frac{\bar{T}^{1}}{T}, \quad z=1+q_{11} \mu, \quad z_{0}=\frac{1}{2},
$$

we conclude that there exists $\delta_{1}>0$ such that

$$
-\beta_{1} \bar{T}_{1}^{*}\left[\frac{\bar{T}^{1}}{T}+\left(1+q_{11} \mu\right) \frac{\bar{T}_{1}^{*} V_{1} T}{T_{1}^{*} \bar{V}_{1} \bar{T}^{1}}+\frac{\bar{V}_{1} T_{1}^{*}}{V_{1} \bar{T}_{1}^{*}}-3\left(1+q_{11} \mu\right)^{1 / 3}\right]<-(L+1)
$$

for all $\frac{T_{1}^{*}}{V_{1}}<\delta_{1}$ and all $\mu \in\left[0, \mu_{1}\right]$. Finally, there exists $\delta_{2}>0$ such that $-\frac{\alpha \eta}{2}+\alpha V_{1}<-\frac{\alpha \eta}{4}$ for all $V_{1}<\delta_{2}$ and all $\mu \in\left[0, \mu_{1}\right]$. Let

$$
\hat{K}_{\delta}=\left\{\left(T, T^{*}, V\right) \in K \cap U \mid T \geq \delta_{0}, V_{1} \geq \delta_{2}, T_{1}^{*} \geq \delta_{1} V_{1}\right\} .
$$

Consider $\left(T, T^{*}, V\right) \in(K \cap U) \backslash \hat{K}_{\delta}$ and let $\mu \in\left[0, \mu_{1}\right]$, then at least one of the following holds:

- $T<\delta_{0}$, in which case $\dot{W} \leq-(L+1)+L \leq-1$;

- $T_{1}^{*} / V_{1}<\delta_{1}$, in which case $\dot{W} \leq-(L+1)+L \leq-1$;

- $V_{1}<\delta_{2}$, in which case $\dot{W} \leq-\frac{\alpha \eta}{4}$; 
Hence, for all $\left(T, T^{*}, V\right) \in(K \cap U) \backslash \hat{K}_{\delta}$ and all $\mu \in\left[0, \mu_{1}\right]$, we have

$$
\dot{W} \leq-\min \left(1, \frac{\alpha \eta}{4}\right)<0 .
$$

We postpone the rest of the proof until we have showed that a similar inequality holds for system (13) - (15).

Case 2: System (13) - (15).

Computing $\dot{W}$ for system (13) - (15), we obtain after some simplifications

$$
\begin{aligned}
\dot{W}= & \left(f(T)-f\left(\bar{T}^{1}\right)\right)\left(1-\frac{\bar{T}^{1}}{T}\right)-\beta_{1} \bar{T}_{1}^{*}\left[\frac{\bar{T}^{1}}{T}+\frac{\bar{T}_{1}^{*} V_{1} T}{T_{1}^{*} \bar{V}_{1} \bar{T}^{1}}+\frac{\bar{V}_{1} T_{1}^{*}}{V_{1} \bar{T}_{1}^{*}}-3\right] \\
& -\sum_{i=2}^{n} k_{i} V_{i}\left(\bar{T}^{i}-\bar{T}^{1}\right)+\mu\left(\frac{V_{1}-\bar{V}_{1}}{V_{1}}\right) \sum_{j=1}^{n} q_{1 j} \frac{N_{j}}{N_{1}} \beta_{j} T_{j}^{*}+\mu \sum_{i=2}^{n} \sum_{j=1}^{n} q_{i j} \frac{N_{j}}{N_{i}} \beta_{j} T_{j}^{*} .
\end{aligned}
$$

Note that the $\mu$ dependent terms can be rearranged as follows:

$$
\mu\left(\sum_{i=1}^{n} q_{i i} \beta_{i} T_{i}^{*}-\frac{\bar{V}_{1}}{V_{1}} \sum_{j=2}^{n} q_{1 j} \frac{N_{j}}{N_{1}} \beta_{j} T_{j}^{*}\right)+\mu\left(\sum_{j=2}^{n} q_{1 j} \frac{N_{j}}{N_{1}} \beta_{j} T_{j}^{*}+\sum_{i=2}^{n} \sum_{j \neq i}^{n} q_{i j} \frac{N_{j}}{N_{i}} \beta_{j} T_{j}^{*}\right)-\mu \frac{\bar{V}_{1}}{V_{1}} q_{11} \beta_{1} T_{1}^{*} .
$$

In the above the first term is non-positive, and the second term can be re-written as follows:

$$
\mu \sum_{i=1}^{n} \alpha_{i} T_{i}^{*}
$$

for suitable $\alpha_{i} \geq 0$, and the third term will be absorbed in the square bracket [ ] term in $\dot{W}$. We find that

$$
\begin{aligned}
\dot{W} \leq & \left(f(T)-f\left(\bar{T}^{1}\right)\right)\left(1-\frac{\bar{T}^{1}}{T}\right)-\beta_{1} \bar{T}_{1}^{*}\left[\frac{\bar{T}^{1}}{T}+\frac{\bar{T}_{1}^{*} V_{1} T}{T_{1}^{*} \bar{V}_{1} \bar{T}^{1}}+\left(1+q_{11} \mu\right) \frac{\bar{V}_{1} T_{1}^{*}}{V_{1} \bar{T}_{1}^{*}}-3\left(1+q_{11} \mu\right)^{1 / 3}\right] \\
& +3 \beta_{1} \bar{T}_{1}^{*}\left(1-\left(1+q_{11} \mu\right)^{1 / 3}\right)+\mu \sum_{i=1}^{n} \alpha_{i} T_{i}^{*}-\sum_{i=2}^{n} k_{i} V_{i}\left(\bar{T}^{i}-\bar{T}^{1}\right) .
\end{aligned}
$$

By Lemma 5, there exist $\eta, \mu_{a}>0$ such that $1^{\prime} V(t)>\eta$ for all $\mu \in\left[0, \mu_{a}\right]$ and all sufficiently large $t$. Let $\alpha=\min _{i \geq 2} k_{i}\left(\bar{T}^{i}-\bar{T}^{1}\right)>0$, then

$$
\sum_{i=2}^{n} k_{i} V_{i}\left(\bar{T}^{i}-\bar{T}^{1}\right) \geq \alpha \sum_{i=2}^{n} V_{i} \geq \alpha\left(\eta-V_{1}\right) .
$$

Thus, by shifting time forward if necessary, we have the inequality

$$
\begin{aligned}
& \dot{W} \leq\left(f(T)-f\left(\bar{T}^{1}\right)\right)\left(1-\frac{\bar{T}^{1}}{T}\right)-\beta_{1} \bar{T}_{1}^{*}\left[\frac{\bar{T}^{1}}{T}+\frac{\bar{T}_{1}^{*} V_{1} T}{T_{1}^{*} \bar{V}_{1} \bar{T}^{1}}+\left(1+q_{11} \mu\right) \frac{\bar{V}_{1} T_{1}^{*}}{V_{1} \bar{T}_{1}^{*}}-3\left(1+q_{11} \mu\right)^{1 / 3}\right] \\
&+3 \beta_{1} \bar{T}_{1}^{*}\left(1-\left(1+q_{11} \mu\right)^{1 / 3}\right)+\mu \sum_{i=1}^{n} \alpha_{i} T_{i}^{*}-\alpha \eta+\alpha V_{1} .
\end{aligned}
$$

Since solutions are in the compact set $K$ for sufficiently large times, there is some $\mu_{a}^{\prime}>0$ such that

$$
\mu \sum_{i=1}^{n} \alpha_{i} T_{i}^{*} \leq \frac{\alpha \eta}{2}, \quad \forall \mu \in\left[0, \mu_{a}^{\prime}\right]
$$

and therefore

$$
\begin{aligned}
& \dot{W} \leq\left(f(T)-f\left(\bar{T}^{1}\right)\right)\left(1-\frac{\bar{T}^{1}}{T}\right)-\beta_{1} \bar{T}_{1}^{*}\left[\frac{\bar{T}^{1}}{T}+\frac{\bar{T}_{1}^{*} V_{1} T}{T_{1}^{*} \bar{V}_{1} \bar{T}^{1}}+\left(1+q_{11} \mu\right) \frac{\bar{V}_{1} T_{1}^{*}}{V_{1} \bar{T}_{1}^{*}}-3\left(1+q_{11} \mu\right)^{1 / 3}\right] \\
&+3 \beta_{1} \bar{T}_{1}^{*}\left(1-\left(1+q_{11} \mu\right)^{1 / 3}\right)-\frac{\alpha \eta}{2}+\alpha V_{1} .
\end{aligned}
$$


Let $\mu_{b}>0$ be such that for all $\mu \in\left[0, \mu_{b}\right]$,

$$
1+q_{11} \mu \in\left[\frac{1}{2}, 1\right], \quad 3 \beta_{1} \bar{T}_{1}^{*}\left(1-\left(1+q_{11} \mu\right)^{1 / 3}\right)-\frac{\alpha \eta}{2} \leq-\frac{\alpha \eta}{4} .
$$

Let $\mu_{1}=\min \left(\mu_{a}, \mu_{a}^{\prime}, \mu_{b}\right)$ and choose sufficiently large $L>0$ so that

$$
3 \beta_{1} \bar{T}_{1}^{*}\left(1-\left(1+q_{11} \mu\right)^{1 / 3}\right)-\alpha \eta+\alpha V_{1}<L
$$

for all solutions of (13) $-(15)$ in $K$ and all $\mu \in\left[0, \mu_{1}\right]$. For any $\mu \in\left[0, \mu_{1}\right]$, we have that

$$
\begin{aligned}
\dot{W} \leq & \left(f(T)-f\left(\bar{T}^{1}\right)\right)\left(1-\frac{\bar{T}^{1}}{T}\right)-\beta_{1} \bar{T}_{1}^{*}\left[\frac{\bar{T}^{1}}{T}+\frac{\bar{T}_{1}^{*} V_{1} T}{T_{1}^{*} \bar{V}_{1} \bar{T}^{1}}+\left(1+q_{11} \mu\right) \frac{\bar{V}_{1} T_{1}^{*}}{V_{1} \bar{T}_{1}^{*}}-3\left(1+q_{11} \mu\right)^{1 / 3}\right] \\
& -\frac{\alpha \eta}{4}+\alpha V_{1},
\end{aligned}
$$

where the first two terms are non-positive and $1+q_{11} \mu \in\left[\frac{1}{2}, 1\right]$. Inspecting the first term in $\dot{W}$, we find that there exists $\delta_{0}>0$ such that

$$
\left(f(T)-f\left(\bar{T}^{1}\right)\left(1-\frac{\bar{T}^{1}}{T}\right)<-(L+1)\right.
$$

for all $T<\delta_{0}$ and all $\mu \in\left[0, \mu_{1}\right]$. Inspecting the second term in $\dot{W}$, we use Lemma 6 with

$$
x=\left(1+q_{11} \mu\right) \frac{\bar{V}_{1} T_{1}^{*}}{V_{1} \bar{T}_{1}^{*}}, \quad y=\frac{\bar{T}^{1}}{T}, \quad z=1+q_{11} \mu, \quad z_{0}=\frac{1}{2},
$$

and conclude that there exists $\delta_{1}>0$ such that

$$
-\beta_{1} \bar{T}_{1}^{*}\left[\frac{\bar{T}^{1}}{T}+\frac{\bar{T}_{1}^{*} V_{1} T}{T_{1}^{*} \bar{V}_{1} \bar{T}^{1}}+\left(1+q_{11} \mu\right) \frac{\bar{V}_{1} T_{1}^{*}}{V_{1} \bar{T}_{1}^{*}}-3\left(1+q_{11} \mu\right)^{1 / 3}\right]<-(L+1)
$$

for all $\frac{T_{1}^{*}}{V_{1}}<\delta_{1}$ and all $\mu \in\left[0, \mu_{1}\right]$. Finally, there exists $\delta_{2}>0$ such that $-\frac{\alpha \eta}{4}+\alpha V_{1}<-\frac{\alpha \eta}{8}$ for all $V_{1}<\delta_{2}$ and all $\mu \in\left[0, \mu_{1}\right]$. Let

$$
\hat{K}_{\delta}=\left\{\left(T, T^{*}, V\right) \in K \cap U \mid T \geq \delta_{0}, V_{1} \geq \delta_{2}, T_{1}^{*} \geq \delta_{1} V_{1}\right\} .
$$

Consider $\left(T, T^{*}, V\right) \in(K \cap U) \backslash \hat{K}_{\delta}$ and let $\mu \in\left[0, \mu_{1}\right]$, then at least one of the following holds:

- $T<\delta_{0}$, in which case $\dot{W} \leq-(L+1)+L \leq-1$;

- $T_{1}^{*} / V_{1}<\delta_{1}$, in which case $\dot{W} \leq-(L+1)+L \leq-1$;

- $V_{1}<\delta_{2}$, in which case $\dot{W} \leq-\frac{\alpha \eta}{8}$;

Hence, for all $\left(T, T^{*}, V\right) \in(K \cap U) \backslash \hat{K}_{\delta}$ and all $\mu \in\left[0, \mu_{1}\right]$, we have

$$
\dot{W} \leq-\min \left(1, \frac{\alpha \eta}{8}\right)<0 .
$$

The remainder of the proof is the same for both of the above two cases and presented next.

The non-negative function $W\left(T, T^{*}, V, \mu\right)$ is continuous and bounded from above on the set $\hat{K}_{\delta} \times\left[0, \mu_{1}\right]$ because $T, T_{1}^{*}, V_{1}$ are bounded away from zero. Hence it attains a finite positive maximum

$$
w:=\max _{\hat{K}_{\delta} \times\left[0, \mu_{1}\right]} W\left(T, T^{*}, V, \mu\right)>0 .
$$

Define a new set

$$
K_{\delta}=\left\{\left(T, T^{*}, V\right) \in K \cap U \mid W\left(T, T^{*}, V, \mu\right) \leq w, \forall \mu \in\left[0, \mu_{1}\right]\right\} .
$$

By construction, we have that $\hat{K}_{\delta} \subset K_{\delta} \subset K \cap U$. The continuity of $W$ implies that $K_{\delta}$ is closed, and therefore compact in $U$. 
It remains to show that all solutions of (10) - (12) in $U$ enter and remain in $K_{\delta}$ for all sufficiently large times. Since $K \cap U$ is an absorbing set for all $\mu \geq 0$ (Lemma 1), without loss of generality we need to prove this for all solutions in $K \cap U$.

Let $\Phi(t)=\left(T(t), T^{*}(t), V(t)\right) \in K \cap U$ be a solution of (10) - (12) for some fixed $\mu \in\left[0, \mu_{1}\right]$. Observe that in the set $(K \cap U) \backslash K_{\delta}$, the inequality $\dot{W} \leq-\min \left(1, \frac{\alpha \eta}{8}\right)<0$ holds. Since $W \geq 0$, there exists $t_{0} \geq 0$ such that $\Phi\left(t_{0}\right) \in \hat{K}_{\delta} \subset K_{\delta}$. We will show that $\Phi(t) \in K_{\delta}$ for all $t \geq t_{0}$. For the sake of contradiction, let us suppose that there exists $t_{1}>t_{0}$ such that $\Phi\left(t_{1}\right) \notin K_{\delta}$. Then there exists $t_{2} \in\left[t_{0}, t_{1}\right)$ such that $\Phi\left(t_{2}\right) \in K_{\delta}$ and $\Phi(t) \notin K_{\delta}$ for all $t \in\left(t_{2}, t_{1}\right]$. On the one hand, we have that

$$
W\left(\Phi\left(t_{2}\right), \mu\right) \leq w<W\left(\Phi\left(t_{1}\right), \mu\right)
$$

by definition of $K_{\delta}$. On the other hand, for all $t \in\left(t_{2}, t_{1}\right]$, we have $\Phi(t) \notin K_{\delta}$ and consequently $\Phi(t) \notin \hat{K}_{\delta}$ so that $\frac{d}{d t} W(\Phi(t), \mu)=\dot{W}<0$. This contradiction shows that $\Phi(t) \in K_{\delta}$ for all $t \geq t_{0}$ and concludes the proof of the Theorem.

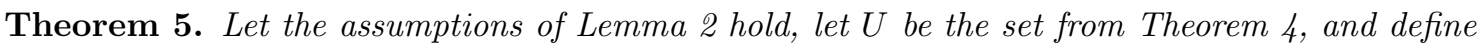

$$
U^{\prime}=\left\{\left(T, T^{*}, V\right) \in \mathbb{R}_{+}^{2 n+1} \mid T_{1}^{*}+V_{1}>0\right\} \supset U .
$$

Then there exist $\mu_{0}>0$ and a continuous map $E:\left[0, \mu_{0}\right] \rightarrow U$ such that

1. $E(0)=E_{1}$ (where $E_{1}$ is the same as in Lemma Q2), and $E(\mu)$ is an equilibrium of (10) - (12) or of (13) - (15) for all $\mu \in\left[0, \mu_{0}\right]$;

2. For each $\mu \in\left[0, \mu_{0}\right], E(\mu)$ is a globally asymptotically stable equilibrium of (10) - (12) or of (13) - (15) in $U^{\prime}$.

Proof. To prove the first assertion, we begin by noting that for $\mu=0, E_{1}$ is a stable hyperbolic equilibrium of (10) - (12) or of (13) - (15) by Lemma 2. Since the vector field of (10) - (12) and (13) - (15) is linear in $\mu$, by the Implicit Function Theorem there exist $h>0$ and a continuous map $E:(-h, h) \rightarrow \mathbb{R}^{2 n+1}$ such that $E(\mu)$ is an equilibrium of (10) - (12) or (13) - (15) for all $\mu \in(-h, h)$. The fact that $E(\mu) \in U$ for all $\mu \in[0, h)$ follows from Proposition 2 and the fact that $\bar{T}_{1}<\bar{T}_{i}, i \geq 2$. Note that for $\mu>0, E(\mu)$ may be positive (if $Q$ is irreducible) or non-negative (if $Q$ is reducible). Nevertheless, in both cases, $\mu>0$ implies $E(\mu) \in U$.

The proof of the second assertion is based on the result of Smith and Waltman (Corollary 2.3 in [14]). We have already established the fact that $E(0)$ is a stable hyperbolic equilibrium of (10) - (12) or (13) - (15). By Theorem $2 E(0)$ is globally asymptotically stable in $U^{\prime}$ for $\mu=0$. In addition, by Theorem 4 there exist

$\mu_{0}>0$ and a compact set $K_{\delta} \subset U$ such that for each $\mu \in\left[0, \mu_{0}\right]$, and each solution $\left(T(t), T^{*}(t), V(t)\right)$ of (10) - (12) or (13) - (15) in $U$, there exists $t_{0}>0$ such that $\left(T(t), T^{*}(t), V(t)\right) \in K_{\delta}$ for all $t>t_{0}$. Hence, the condition (H1) of Corollary 2.3 in [14] holds. The Proposition 2.3 itself then implies the global stability of $E(\mu)$ in $U$ for all sufficiently small $\mu \geq 0$. Finally, solutions of (10) - (12) or (13) - (15) starting in $U^{\prime}$ enter $U$ instantaneously, hence global stability of $E(\mu)$ in $U^{\prime}$ follows as well.

\section{Appendix: Inclusion of loss of virus in the model}

\section{Single-strain}

When taking the loss of the virus particle upon infection into account, model (11) becomes

$$
\begin{aligned}
\dot{T} & =f(T)-k V T \\
\dot{T}^{*} & =k V T-\beta T^{*} \\
\dot{V} & =N \beta T^{*}-\gamma V-k V T,
\end{aligned}
$$

We still assume that the growth rate of the healthy cell population is given by (2), hence $E_{0}=\left(T_{0}, 0,0\right)$ is still an equilibrium of (37). A second, positive equilibrium may exist if the following quantities are positive:

$$
\bar{T}=\frac{\gamma}{k(N-1)}, \quad \bar{T}^{*}=\frac{f(\bar{T})}{\beta}, \quad \bar{V}=\frac{f(\bar{T})}{k \bar{T}} .
$$


Note that this is the case iff $N>1$ and $f\left(\frac{\gamma}{k(N-1)}\right)>0$, or equivalently by (2) that $\bar{T}=\frac{\gamma}{k(N-1)}<T_{0}$. In terms of the basic reproduction number

$$
\mathcal{R}^{0}:=\frac{k(N-1)}{\gamma} T_{0}=\frac{T_{0}}{\bar{T}},
$$

existence of a positive equilibrium is therefore equivalent to $\mathcal{R}^{0}>1$. Assuming that $\mathcal{R}^{0}>1$, we will still denote this disease steady state by $E=\left(\bar{T}, \bar{T}^{*}, \bar{V}\right)$. We introduce the following condition.

$$
\left(\mathbf{C}^{\prime}\right) f^{\prime}(c)+\frac{k}{\gamma} f(\bar{T}) \leq 0 \text {, for all } c \in\left[0, T_{0}\right] .
$$

Note that this condition is satisfied when $f(T)$ is a decreasing function with sufficiently large negative derivative.

Theorem 6. Let $\left(\mathbf{C}^{\prime}\right)$ hold. Then the equilibrium $E$ is globally asymptotically stable for (37) with respect to initial conditions satisfying $T^{*}(0)+V(0)>0$.

Proof. Consider the following function on $\operatorname{int}\left(\mathbb{R}_{+}^{3}\right)$ :

$$
W=(N-1) \int_{\bar{T}}^{T}\left(1-\frac{\bar{T}}{\tau}\right) d \tau+N \int_{\bar{T}^{*}}^{T^{*}}\left(1-\frac{\bar{T}^{*}}{\tau}\right) d \tau+\int_{\bar{V}}^{V}\left(1-\frac{\bar{V}}{\tau}\right) d \tau .
$$

Then

$$
\begin{aligned}
\dot{W} & =(N-1)(f(T)-k V T)\left(1-\frac{\bar{T}}{T}\right)+N\left(k V T-\beta T^{*}\right)\left(1-\frac{\bar{T}^{*}}{T^{*}}\right)+\left(N \beta T^{*}-\gamma V-k V T\right)\left(1-\frac{\bar{V}}{V}\right) \\
& =(N-1) f(T)\left(1-\frac{\bar{T}}{T}\right)-N k V T \frac{\bar{T}^{*}}{T^{*}}+N \beta \bar{T}^{*}-N \beta T^{*} \frac{\bar{V}}{V}+\gamma \bar{V}+k \bar{V} T \\
& =(N-1)(f(T)-f(\bar{T}))\left(1-\frac{\bar{T}}{T}\right)+(N-1) f(\bar{T})\left(1-\frac{\bar{T}}{T}\right)+N \beta \bar{T}^{*}\left[2-\frac{V T \bar{T}^{*}}{\bar{V} \bar{T} T^{*}}-\frac{T^{*} \bar{V}}{\bar{T} * V}\right]-\beta \bar{T}^{*}+\beta \bar{T}^{*} \frac{T}{\bar{T}} \\
& =(N-1)(f(T)-f(\bar{T}))\left(1-\frac{\bar{T}}{T}\right)+(N-1) \beta \bar{T}^{*}\left(1-\frac{\bar{T}}{T}\right)+N \beta \bar{T}^{*}\left[2-\frac{V T \bar{T}^{*}}{\bar{V} \bar{T} T^{*}}-\frac{T^{*} \bar{V}}{\bar{T} * V}\right]-\beta \bar{T}^{*}+\beta \bar{T}^{*} \frac{T}{\bar{T}} \\
& =(N-1)(f(T)-f(\bar{T}))\left(1-\frac{\bar{T}}{T}\right)+\beta \bar{T}^{*}\left(-2+\frac{\bar{T}}{T}+\frac{T}{\bar{T}}\right)+N \beta \bar{T}^{*}\left[3-\frac{V T \bar{T}^{*}}{\bar{V} \bar{T} T^{*}}-\frac{T^{*} \bar{V}}{\bar{T} * V}-\frac{\bar{T}}{T}\right] \\
& =\left[(N-1)(f(T)-f(\bar{T})) \bar{T}+\beta \bar{T}^{*}(T-\bar{T})\right] \frac{(T-\bar{T})}{T \bar{T}}+N \beta \bar{T}^{*}\left[3-\frac{V T \bar{T}^{*}}{\bar{V} \bar{T} T^{*}}-\frac{T^{*} \bar{T}}{\bar{T} * V}-\frac{\bar{T}}{T}\right]
\end{aligned}
$$

where we used (38) repeatedly; in particular in the second, third and fourth equation. By the mean value theorem there is some $c \in(T, \bar{T})$ or $(\bar{T}, T)$ such that

$$
f(T)-f(\bar{T})=f^{\prime}(c)(T-\bar{T}),
$$

hence using (38) once more

$$
\dot{W}=(N-1)\left[f^{\prime}(c)+\frac{k}{\gamma} f(\bar{T})\right] \frac{(T-\bar{T})^{2}}{T}+N \beta \bar{T}^{*}\left[3-\frac{V T \bar{T}^{*}}{\bar{V} \bar{T} T^{*}}-\frac{T^{*} \bar{V}}{\bar{T}^{*} V}-\frac{\bar{T}}{T}\right] .
$$

The first term is non-positive by $\left(\mathbf{C}^{\prime}\right)$ and because we can assume that $T \leq T_{0}$ by dissipativity (see Lemma 7later). The second term is non-positive as well since the geometric mean of 3 non-negative numbers is not larger than the arithmetic mean of those numbers. We conclude that $\dot{W} \leq 0$ in int $\left(\mathbb{R}_{+}^{3}\right)$, hence local stability of $E$ follows. Notice that $\dot{W}$ equals zero if and only if both the first term and the second term are zero, This happens at points where:

$$
\frac{\bar{T}}{T}=1 \text { and } \frac{\bar{T}^{*} V}{T^{*} \bar{V}}=1
$$

Then LaSalle's Invariance Principle implies that all bounded solutions (and as before, solutions are easily shown to be bounded, see also Lemma 7 later) in $\operatorname{int}\left(\mathbb{R}_{+}^{3}\right)$ converge to the largest invariant set in

$$
M=\left\{\left(T, T^{*}, V\right) \in \operatorname{int}\left(\mathbb{R}_{+}^{3}\right) \mid \frac{\bar{T}}{T}=1, \frac{\bar{T}^{*} V}{T^{*} \bar{V}}=1\right\} .
$$


It is clear that the largest invariant set in $M$ is the singleton $\{E\}$. Finally, note that forward solutions starting on the boundary of $\mathbb{R}_{+}^{3}$ with either $T_{1}(0)$ or $V_{1}(0)$ positive, enter int $\left(\mathbb{R}_{+}^{3}\right)$ instantaneously. This concludes the proof.

\section{Competitive exclusion}

Now we modify the multi-strain model (44) - (6) to

$$
\begin{aligned}
\dot{T} & =f(T)-k V T, \quad T \in \mathbb{R}_{+} \\
\dot{T}^{*} & =K V T-B T^{*}, \quad T^{*} \in \mathbb{R}_{+}^{n} \\
\dot{V} & =\hat{N} B T^{*}-\Gamma V-K V T, \quad V \in \mathbb{R}_{+}^{n},
\end{aligned}
$$

where $k=\left(k_{1}, \ldots, k_{n}\right), K=\operatorname{diag}\left(k_{1}, \ldots, k_{n}\right), B=\operatorname{diag}\left(\beta_{1}, \ldots, \beta_{n}\right), \hat{N}=\operatorname{diag}\left(N_{1}, \ldots, N_{n}\right)$, and $\Gamma=\operatorname{diag}\left(\gamma_{1}, \ldots, \gamma_{n}\right)$. Suppose that each strain is capable to persist at steady state by itself, that is, $N_{i}>1$ and $\bar{T}_{i}=\frac{\gamma_{i}}{k_{i}\left(N_{i}-1\right)}<T_{0}$ and denote the corresponding equilibria also by $E_{1}, \ldots, E_{n}$. Assume that

$$
0<\bar{T}_{1} \leq \bar{T}_{2} \leq \ldots \leq \bar{T}_{n}<T_{0} .
$$

In addition, suppose that $\left(\mathbf{C}^{\prime}\right)$ holds with $\bar{T}=\bar{T}_{1}$. Then we have the following.

Theorem 7. The single strain equilibrium $E_{1}$ is globally asymptotically stable for (39) - 41) with respect to initial conditions satisfying $T_{1}^{*}(0)+V_{1}(0)>0$.

Proof. Consider the function $W$ defined on $U:=\left\{\left(T, T^{*}, V\right) \in \mathbb{R}^{2 n+1} \mid T, T_{1}^{*}, V_{1}>0\right\}$ as

$$
\begin{aligned}
W= & \left(N_{1}-1\right) \int_{\bar{T}_{1}}^{T}\left(1-\frac{\bar{T}_{1}}{\tau}\right) d \tau+N_{1} \int_{\bar{T}_{1}^{*}}^{T_{1}^{*}}\left(1-\frac{\bar{T}_{1}^{*}}{\tau}\right) d \tau+\int_{\bar{V}_{1}}^{V_{1}}\left(1-\frac{\bar{V}_{1}}{\tau}\right) d \tau \\
& +\sum_{i=2}^{n} \frac{N_{1}-1}{N_{i}-1}\left(N_{i} T_{i}^{*}+V_{i}\right) .
\end{aligned}
$$

Computing $\dot{W}$, we find that

$$
\begin{aligned}
\dot{W}= & \left(N_{1}-1\right)\left[f^{\prime}(c)+\frac{k}{\gamma} f\left(\bar{T}_{1}\right)\right] \frac{\left(T-\bar{T}_{1}\right)^{2}}{T}+N_{1} \beta_{1} \bar{T}_{1}^{*}\left[3-\frac{V_{1} T \bar{T}_{1}^{*}}{\bar{V}_{1} \bar{T}_{1} T_{1}^{*}}-\frac{T_{1}^{*} \bar{V}_{1}}{\bar{T}_{1}^{*} V_{1}}-\frac{\bar{T}_{1}}{T}\right] \\
& +\sum_{i=2}^{n}\left(-k_{i} V_{i}\left(T-\bar{T}_{1}\right)+\frac{\left(N_{1}-1\right)}{N_{i}-1}\left(N_{i} k_{i} V_{i} T-N_{i} \beta_{i} T_{i}^{*}+N_{i} \beta_{i} T_{i}^{*}-\gamma_{i}-k_{i} V_{i} T\right)\right) .
\end{aligned}
$$

After simplifications, we have

$$
\begin{aligned}
\dot{W}= & \left(N_{1}-1\right)\left[f^{\prime}(c)+\frac{k}{\gamma} f\left(\bar{T}_{1}\right)\right] \frac{\left(T-\bar{T}_{1}\right)^{2}}{T}+N_{1} \beta_{1} \bar{T}_{1}^{*}\left[3-\frac{V_{1} T \bar{T}_{1}^{*}}{\bar{V}_{1} \bar{T}_{1} T_{1}^{*}}-\frac{T_{1}^{*} \bar{V}_{1}}{\bar{T}_{1}^{*} V_{1}}-\frac{\bar{T}_{1}}{T}\right] \\
& -\left(N_{1}-1\right) \sum_{i=2}^{n} k_{i} V_{i}\left(\bar{T}_{i}-\bar{T}_{1}\right) .
\end{aligned}
$$

The first term is non-positive since $\left(\mathbf{C}^{\prime}\right)$ with $\bar{T}=\bar{T}_{1}$ holds and because $T \leq T_{0}$ by disspiativity (see Lemma 7 later). The second term is non-positive is well, and so is the third by (42). Thus $\dot{W} \leq 0$ which already implies that $E_{1}$ is stable. An application of LaSalle's Invariance Principle shows that all bounded solutions in $U$ (boundedness follows from Lemma 7 which is proved later) converge to the largest invariant set in

$$
\left\{\left(T, T_{1}^{*}, \ldots, T_{n}^{*}, V_{1}, \ldots, V_{n}\right) \in U \mid \frac{\bar{T}^{1}}{T}=1, \frac{\bar{T}_{1}^{*} V_{1}}{T_{1}^{*} \bar{V}_{1}}=1, \quad V_{i}=0, \quad i>2\right\},
$$

which is easily shown to be the singleton $\left\{E_{1}\right\}$. Finally, solutions on the boundary of $U$ with $T_{1}^{*}(0)+V_{1}(0)>0$ enter $U$ instantaneously, which concludes the proof. 


\section{Adding mutations}

We modify the model (39) - (41) to account for mutations. Again, we consider two alternative models

$$
\begin{aligned}
\dot{T} & =f(T)-k V T, \quad T \in \mathbb{R}_{+} \\
\dot{T}^{*} & =P(\mu) K V T-B T^{*}, \quad T^{*} \in \mathbb{R}_{+}^{n} \\
\dot{V} & =\hat{N} B T^{*}-\Gamma V-K V T, \quad V \in \mathbb{R}_{+}^{n},
\end{aligned}
$$

and

$$
\begin{aligned}
\dot{T} & =f(T)-k V T, \quad T \in \mathbb{R}_{+} \\
\dot{T}^{*} & =K V T-B T^{*}, \quad T^{*} \in \mathbb{R}_{+}^{n} \\
\dot{V} & =P(\mu) \hat{N} B T^{*}-\Gamma V-K V T, \quad V \in \mathbb{R}_{+}^{n},
\end{aligned}
$$

where $k, K, B, \hat{N}, \Gamma$ are the same as before, and $P(\mu)=I+\mu Q$ and $Q$ is a stochastic matrix with non-negative off-diagonal entries.

Lemma 7. Both systems (43) and (44) are dissipative, i.e. there is some compact set $K$ such that every solution eventually enters $K$ and remains in $K$ forever after.

Proof. The proof is similar to the proof of Lemma 1 and will be omitted.

Lemma 8. For $\mu=0$, let all single strain equilibria $E_{1}, E_{2} \ldots, E_{n}$ exist for either (43) or (44), and assume that

$$
\bar{T}^{1}<\bar{T}^{2}<\cdots<\bar{T}^{n}<\bar{T}^{n+1}:=T_{0},
$$

and

$$
f^{\prime}\left(\bar{T}^{j}\right) \leq 0, \text { for all } j=1, \ldots, n+1 .
$$

Then the Jacobian matrices of (43) or (44), evaluated at any of the $E_{i}$ 's, $i=1, \ldots, n+1$ (where $E_{n+1}:=E_{0}$ ) have the following properties: $J\left(E_{i}\right)$ has $i-1$ eigenvalues (counting multiplicities) in the open right half plane and $2(n+1)-i$ eigenvalues in the open left half plane. In particular, $J\left(E_{1}\right)$ is Hurwitz.

Proof. The proof is similar to that of Lemma2. The only difference is that the entries of the Jacobian matrices change. In particular, the $(3,1)$ and $(3,3)$ entry of $A_{1}^{i}$ now become $-k_{i} \bar{V}_{i}$ and $-\gamma_{i}-k \bar{T}^{i}$ respectively, but by (45) and Lemma 3.4 in [1], $A_{1}^{i}$ is still Hurwitz.

To study equilibria of systems (43) and (44), we introduce the matrix

$$
A(\mu)=\Gamma^{-1}(\hat{N} P(\mu)-I) K,
$$

which has non-negative off-diagonal entries for $\mu>0$ and

$$
A(0)=\operatorname{diag}\left(\frac{k_{1}\left(N_{1}-1\right)}{\gamma_{1}}, \ldots, \frac{k_{n}\left(N_{n}-1\right)}{\gamma_{n}}\right)=\operatorname{diag}\left(\frac{1}{\bar{T}_{1}}, \ldots, \frac{1}{\bar{T}_{n}}\right) .
$$

Clearly, Proposition 1 holds with $A(\mu)$ given by (47). Hence, we have the following.

Proposition 3. Let the assumptions of Lemma 8 hold and suppose that the strains are renumbered so that $A(\mu)$ has the form (23). Let $E_{j}(\mu)=\left(\hat{T}_{j}(\mu), \hat{T}_{j}^{*}(\mu), \hat{V}_{j}(\mu)\right)$ denote the nontrivial equilibria of both (43) and (44) for small $\mu>0$. Then

1. $E_{j}(\mu)$ is positive if and only if $\frac{1}{\hat{T}_{j}(\mu)}$ is an eigenvalue of $A(\mu)$ with a positive eigenvector $V_{j}$.

2. $E_{j}(\mu)$ is non-negative if and only if $\frac{1}{\hat{T}_{j}(\mu)}$ is an eigenvalue of $A(\mu)$ with a non-negative eigenvector $V_{j}$.

3. $E_{j}(\mu) \notin \mathbb{R}_{+}^{2 n+1}$ if and only if $\frac{1}{\hat{T}_{j}(\mu)}$ is an eigenvalue of $A(\mu)$ with eigenvector $V_{j}$ which is not signdefinite. 
Proof. We will prove the Proposition only for system (43) (the proof for (44) is similar). Observe that at equilibrium, $\hat{T}_{j}^{*}(\mu)=(\hat{N} B)^{-1}\left(\Gamma+K \hat{T}_{j}(\mu)\right) \hat{V}_{j}(\mu)$. Hence, the the signs of the corresponding components of $\hat{T}_{j}^{*}(\mu)$ and $\hat{V}_{j}(\mu)$ are the same. Substituting the above expression into (43), we find that $\hat{V}_{j}(\mu)$ must satisfy

$$
\left[\Gamma^{-1}(\hat{N} P(\mu)-I) K-\frac{1}{\hat{T}_{j}(\mu)} I\right] \hat{V}_{j}(\mu)=\left[A(\mu)-\frac{1}{\hat{T}_{j}(\mu)} I\right] \hat{V}_{j}(\mu)=0 .
$$

Thus for each nontrivial equilibrium $E_{j}(\mu)$, the quantity $\frac{1}{\hat{T}_{j}(\mu)}$ must be an eigenvalue of $A(\mu)$ and $\hat{V}_{j}(\mu)$ must be a multiple of the corresponding eigenvector $V_{j}$. If $V_{j}$ is not sign definite, it follows that $E_{j}(\mu) \notin \mathbb{R}_{+}^{2 n+1}$. For all $V_{j} \geq 0$, the components of $E_{j}(\mu)$ are uniquely determined via

$$
\hat{V}_{j}(\mu)=\frac{f\left(\hat{T}_{j}(\mu)\right)}{\hat{T}_{j}(\mu) k^{\prime} V_{j}} V_{j}, \quad \hat{T}_{j}^{*}(\mu)=(\hat{N} B)^{-1}\left(\Gamma+K \hat{T}_{j}(\mu)\right) \hat{V}_{j}(\mu) .
$$

Hence $E_{j}(\mu)$ is positive (non-negative) if and only if $V_{j}$ is positive (non-negative).

\section{Lower bounds}

Lemma 9. Suppose that (45) holds. Then there exist $\eta, \mu_{0}>0$ such that

$$
\liminf _{t \rightarrow \infty} 1^{\prime} V(t) \geq \eta>0
$$

for any $\mu \in\left[0, \mu_{0}\right]$ and for any solution of (43) and (44) with $1^{\prime} V(t)>0$.

Proof. We will prove the claim for system (43) (the proof for (44) is similar). The proof consists of two parts. We first show that there exist $\eta_{0}, \mu_{0}>0$ such that $\liminf _{t \rightarrow \infty} 1^{\prime}\left(T^{*}(t)+V(t)\right) \geq \eta_{0}>0$ for all solutions with $T^{*}(t), V(t) \neq 0$. We choose $n$ positive numbers $\tilde{N}_{i}$ so that $\frac{\gamma_{i}+k_{i} T_{0}}{k_{i} T_{0}}<\tilde{N}_{i}<N_{i}$ for all $1 \leq i \leq n$. This is possible since we assume $\bar{T}_{i}=\frac{\gamma_{i}}{k_{i}\left(N_{i}-1\right)}<T_{0}$ which is equivalent to $N_{i}>\frac{\gamma_{i}+k_{i} T_{0}}{k_{i} T_{0}}$. Let $v=(\tilde{N}, 1)$. It follows that

$v^{\prime}\left(\begin{array}{cc}-B & K T_{0} \\ \hat{N} B & -\Gamma-K T_{0}\end{array}\right)=\left(b_{1}\left(N_{1}-\tilde{N}_{1}\right), \ldots, b_{n}\left(N_{n}-\tilde{N}_{n}\right), k_{1} T_{0} \tilde{N}_{1}-\left(\gamma_{1}+k_{1} T_{0}\right), \ldots, k_{n} T_{0} \tilde{N}_{n}-\left(\gamma_{n}+k_{n} T_{0}\right)\right)$

is a positive vector. By continuity, there exists a $\mu_{0}>0$ such that

$$
v^{\prime} M\left(T_{0}, \mu\right), \quad \text { where } M(T, \mu):=\left(\begin{array}{cc}
-B & P(\mu) K T \\
\hat{N} B & -\Gamma-K T
\end{array}\right)
$$

is a positive vector for all $\mu \in\left[0, \mu_{0}\right]$.

Consider a system

$$
\begin{aligned}
\dot{T} & =f(T)-k^{\prime} V T, T \in \mathbb{R}_{+} \\
\dot{T}^{*} & =P(\mu) K V T-B T^{*}, T^{*} \in \mathbb{R}_{+}^{n}, \\
\dot{V} & =\hat{N} B T^{*}-\Gamma V-K V T, V \in \mathbb{R}_{+}^{n}, \\
\dot{\mu} & =0, \mu \in\left[0, \mu_{0}\right] .
\end{aligned}
$$

Let $K^{\prime}$ be the forward invariant compact set for (43) established in Lemma 7 and define $K=K^{\prime} \times\left[0, \mu_{0}\right]$. It is clear that $K$ is compact and forward invariant under (48) - (51) The set $K_{0}=\left(\left[0, T_{0}\right] \times 0 \times 0 \times\left[0, \mu_{0}\right]\right) \cap K$ is clearly a compact forward invariant subset of $K$.

Let $\Pi\left(T^{*}, V\right):=v^{\prime}\left(T^{*}, V\right)$. The function $\Pi$ is clearly smooth, zero on $K_{0}$, and positive on $K \backslash K_{0}$. Furthermore,

$$
\frac{\dot{\Pi}}{\Pi}=\psi:=\frac{v^{\prime} M(T, \mu)\left(T^{*}, V\right)}{v^{\prime}\left(T^{*}, V\right)}
$$

is lower semi-continuous on $K$ by Lemma 4 once we define the value of $\psi$ on $K_{0}$ as

$$
\psi(T, \mu)=\min _{i=1, \ldots, n} \frac{v^{\prime} M(T, \mu)_{i}}{v_{i}} .
$$


We note that the function $\psi(T, \mu)$ is continuous in $(T, \mu)$. Since all solutions of (48) - (51) in $K_{0}$ have the property that $\lim _{t \rightarrow \infty} T(t)=T_{0}$, it implies that $\psi(T(t), \mu)>0$ for all sufficiently large $t$. Hence by Theorem 3, the set $K_{0}$ is a uniform strong repellor in $K$. If we use the $L^{1}$-norm of $\left(T^{*}, V\right)$ as the distance function to $K_{0}$, we find that there exists an $\eta_{0}>0$ such that

$$
\liminf _{t \rightarrow \infty} 1^{\prime}\left(T^{*}+V\right) \geq \eta_{0}
$$

for all solutions of (48) - (51) in $K \backslash K_{0}$.

To complete the proof, we need to show that there exists $\eta>0$ such that $\liminf _{t \rightarrow \infty} 1^{\prime} V(t) \geq \eta>0$ for all solutions with $1^{\prime} V(t)>0$. Observe that $1^{\prime} V(t)>0$ implies that $1^{\prime} T^{*}(t)>0$. Hence by the result of part one, we have that $\liminf _{t \rightarrow \infty} 1^{\prime}\left(T^{*}(t)+V(t)\right) \geq \eta_{0}>0$, or equivalently, $1^{\prime} T^{*}(t)>\eta_{0} / 2-1^{\prime} V(t)$ for all sufficiently large $t$. From (50), we have that

$$
1^{\prime} \dot{V} \geq \sum_{i=1}^{n} N_{i} \beta_{i} T_{i}^{*}-\sum_{i=1}^{n}\left(\gamma_{i}+k_{i} T\right) V_{i} \geq \sum_{i=1}^{n} N_{i} \beta_{i} T_{i}^{*}-\sum_{i=1}^{n}\left(\gamma_{i}+k_{i} T_{0}\right) V_{i} .
$$

Hence,

$$
1^{\prime} \dot{V} \geq A_{0}\left(\frac{\eta_{0}}{2}-1^{\prime} V(t)\right)-A_{1} 1^{\prime} V(t), \quad A_{0}:=\min _{i}\left(N_{i} \beta_{i}\right)>0, A_{1}:=\max _{i}\left(\gamma_{i}+k_{i} T_{0}\right)>0
$$

holds for large $t$. It follows immediately that

$$
\liminf _{t \rightarrow \infty} 1^{\prime} V(t) \geq \eta=\frac{\eta_{0} A_{0}}{2\left(A_{0}+A_{1}\right)}>0 .
$$

\section{Existence of absorbing compact set for small $\mu>0$.}

Theorem 8. Let $K$ be the absorbing compact set established in Lemma 7, and let

$$
U=\left\{\left(T, T^{*}, V\right) \in \mathbb{R}_{+}^{2 n+1} \mid T, T_{1}^{*}, V_{1}>0\right\} .
$$

Suppose that there exists $\epsilon>0$ such that

$$
\left(\mathbf{C}_{\epsilon}\right) f^{\prime}(c)+\frac{k_{1}}{\gamma_{1}} f\left(\bar{T}_{1}\right) \leq-\epsilon<0, \text { for all } c \in\left[0, T_{0}\right] .
$$

Then there exist $\mu_{1}>0$ and a compact set $K_{\delta} \subset U$ such that for any $\mu \in\left[0, \mu_{1}\right]$ and for any solution of system (43) in $U$, there exists a $t_{0}>0$ such that $\left(T(t), T^{*}(t), V(t)\right) \in K_{\delta}$ for all $t>t_{0}$.

An identical statement holds for system (44).

Proof. (a) We first prove the statement for system (43). Consider the function

$$
\begin{aligned}
W= & \left(N_{1}-1\right) \int_{\bar{T}_{1}}^{T}\left(1-\frac{\bar{T}_{1}}{\tau}\right) d \tau+N_{1} \int_{\bar{T}_{1}^{*}}^{T_{1}^{*}}\left(1-\frac{\bar{T}_{1}^{*}}{\tau}\right) d \tau+\int_{\bar{V}_{1}}^{V_{1}}\left(1-\frac{\bar{V}_{1}}{\tau}\right) d \tau \\
& +\sum_{i=2}^{n} \frac{N_{1}-1}{N_{i}-1}\left(N_{i} T_{i}^{*}+V_{i}\right) .
\end{aligned}
$$

Computing $\dot{W}$ for the system (43), we obtain

$$
\begin{aligned}
\dot{W}= & \left(N_{1}-1\right)\left[f^{\prime}(c)+\frac{k}{\gamma} f\left(\bar{T}_{1}\right)\right] \frac{\left(T-\bar{T}_{1}\right)^{2}}{T}+N_{1} \beta_{1} \bar{T}_{1}^{*}\left[3-\frac{V_{1} T \bar{T}_{1}^{*}}{\bar{V}_{1} \bar{T}_{1} T_{1}^{*}}-\frac{T_{1}^{*} \bar{V}_{1}}{\bar{T}_{1}^{*} V_{1}}-\frac{\bar{T}_{1}}{T}\right] \\
& -\left(N_{1}-1\right) \sum_{i=2}^{n} k_{i} V_{i}\left(\bar{T}_{i}-\bar{T}_{1}\right)+\mu N_{1} \frac{T_{1}^{*}-\bar{T}_{1}^{*}}{T_{1}^{*}} \sum_{j=1}^{n} q_{1 j} k_{j} V_{j} T+\mu\left(N_{1}-1\right) \sum_{i=2}^{n} \frac{N_{i}}{N_{i}-1} \sum_{j=1}^{n} q_{i j} k_{j} V_{j} T,
\end{aligned}
$$


Recombining the terms, we find that

$$
\begin{aligned}
\dot{W}= & \left(N_{1}-1\right)\left[f^{\prime}(c)+\frac{k}{\gamma} f\left(\bar{T}_{1}\right)\right] \frac{\left(T-\bar{T}_{1}\right)^{2}}{T}+N_{1} \beta_{1} \bar{T}_{1}^{*}\left[3-\frac{V_{1} T \bar{T}_{1}^{*}}{\bar{V}_{1} \bar{T}_{1} T_{1}^{*}}-\frac{T_{1}^{*} \bar{V}_{1}}{\bar{T}_{1}^{*} V_{1}}-\frac{\bar{T}_{1}}{T}\right] \\
& -\left(N_{1}-1\right) \sum_{i=2}^{n} k_{i} V_{i}\left(\bar{T}_{i}-\bar{T}_{1}\right)+\mu\left(N_{1}-1\right) \sum_{i=1}^{n} \frac{N_{i}}{N_{i}-1} \sum_{j=1}^{n} q_{i j} k_{j} V_{j} T \\
& -\mu N_{1} q_{11} \frac{\bar{T}_{1}^{*} V_{1} T}{T_{1}^{*}}-\mu N_{1} \frac{\bar{T}_{1}^{*}}{T_{1}^{*}} \sum_{j=1}^{n} q_{1 j} k_{j} V_{j} T,
\end{aligned}
$$

where the last term is clearly non-positive. Let

$$
\begin{aligned}
\alpha & =\left(N_{1}-1\right) \min _{i \geq 2} k_{i}\left(\bar{T}_{i}-\bar{T}_{1}\right)>0, \\
L & =\sup _{K}\left(N_{1}-1\right) \sum_{i=1}^{n} \frac{N_{i}}{N_{i}-1} \sum_{j=1}^{n} q_{i j} k_{j} V_{j} T \geq 0 .
\end{aligned}
$$

By Lemma 9, there exist $\eta, \mu_{a}>0$ such that $1^{\prime} V(t)>\eta$ for all $\mu \in\left[0, \mu_{a}\right]$ and all sufficiently large $t$. Hence, by shifting time forward if necessary, we have the inequality

$$
\begin{aligned}
\dot{W} \leq & -\epsilon\left(N_{1}-1\right) \frac{\left(T-\bar{T}_{1}\right)^{2}}{T}+N_{1} \beta_{1} \bar{T}_{1}^{*}\left[3-\frac{V_{1} T \bar{T}_{1}^{*}}{\bar{V}_{1} \bar{T}_{1} T_{1}^{*}}-\frac{T_{1}^{*} \bar{V}_{1}}{\bar{T}_{1}^{*} V_{1}}-\frac{\bar{T}_{1}}{T}\right] \\
& -\alpha\left(\eta-V_{1}\right)+\mu L-\mu N_{1} q_{11} \frac{\bar{T}_{1}^{*} V_{1} T}{T_{1}^{*}},
\end{aligned}
$$

which holds in $K$ for all $\mu \in\left[0, \mu_{a}\right]$. We combine the second and the last terms to obtain

$$
\begin{aligned}
\dot{W} \leq & -\epsilon\left(N_{1}-1\right) \frac{\left(T-\bar{T}_{1}\right)^{2}}{T}+N_{1} \beta_{1} \bar{T}_{1}^{*}\left[3-\left(1+q_{11} \mu\right) \frac{V_{1} T \bar{T}_{1}^{*}}{\bar{V}_{1} \bar{T}_{1} T_{1}^{*}}-\frac{T_{1}^{*} \bar{V}_{1}}{\bar{T}_{1}^{*} V_{1}}-\frac{\bar{T}_{1}}{T}\right] \\
& -\alpha\left(\eta-V_{1}\right)+\mu L .
\end{aligned}
$$

Further, we rewrite the above inequality as

$$
\begin{aligned}
\dot{W} \leq & -\epsilon\left(N_{1}-1\right) \frac{\left(T-\bar{T}_{1}\right)^{2}}{T}+N_{1} \beta_{1} \bar{T}_{1}^{*}\left[3\left(1+q_{11} \mu\right)^{1 / 3}-\left(1+q_{11} \mu\right) \frac{V_{1} T \bar{T}_{1}^{*}}{\bar{V}_{1} \bar{T}_{1} T_{1}^{*}}-\frac{T_{1}^{*} \bar{V}_{1}}{\bar{T}_{1}^{*} V_{1}}-\frac{\bar{T}_{1}}{T}\right] \\
& -\alpha\left(\eta-V_{1}\right)+\mu L+3 N_{1} \beta_{1} \bar{T}_{1}^{*}\left[1-\left(1+q_{11} \mu\right)^{1 / 3}\right] .
\end{aligned}
$$

Let $\mu_{b}>0$ be such that for all $\mu \in\left[0, \mu_{b}\right]$,

$$
\left(1+q_{11} \mu\right) \in\left[\frac{1}{2}, 1\right], \quad-\alpha \eta+\mu L+3 N_{1} \beta_{1} \bar{T}_{1}^{*}\left[1-\left(1+q_{11} \mu\right)^{1 / 3}\right] \leq-\frac{\alpha \eta}{2} .
$$

Now we let $\mu_{1}=\min \left[\mu_{a}, \mu_{b}\right]$, so that for all $\mu \in\left[0, \mu_{1}\right]$ and all points in $K$,

$$
\begin{aligned}
\dot{W} \leq & -\epsilon\left(N_{1}-1\right) \frac{\left(T-\bar{T}_{1}\right)^{2}}{T}+N_{1} \beta_{1} \bar{T}_{1}^{*}\left[3\left(1+q_{11} \mu\right)^{1 / 3}-\left(1+q_{11} \mu\right) \frac{V_{1} T \bar{T}_{1}^{*}}{\bar{V}_{1} \bar{T}_{1} T_{1}^{*}}-\frac{T_{1}^{*} \bar{V}_{1}}{\bar{T}_{1}^{*} V_{1}}-\frac{\bar{T}_{1}}{T}\right] \\
& -\frac{\alpha \eta}{2}+\alpha V_{1} .
\end{aligned}
$$

Let $L_{1}=\alpha \sup _{K} V_{1}$. Inspecting the first term in $\dot{W}$, we find that there exists $\delta_{0}>0$ such that

$$
-\epsilon\left(N_{1}-1\right) \frac{\left(T-\bar{T}_{1}\right)^{2}}{T}<-L_{1}
$$

for all $T<\delta_{0}$. Similarly, inspecting the second term in $\dot{W}$ and using Lemma 6 , we find that there exists $\delta_{1}>0$ such that

$$
N_{1} \beta_{1} \bar{T}_{1}^{*}\left[3\left(1+q_{11} \mu\right)^{1 / 3}-\left(1+q_{11} \mu\right) \frac{V_{1} T \bar{T}_{1}^{*}}{\bar{V}_{1} \bar{T}_{1} T_{1}^{*}}-\frac{T_{1}^{*} \bar{V}_{1}}{\bar{T}_{1}^{*} V_{1}}-\frac{\bar{T}_{1}}{T}\right]<-L_{1}
$$


for all $\frac{T_{1}^{*}}{V_{1}}<\delta_{1}$ and all $\mu \in\left[0, \mu_{1}\right]$. Finally, there exists $\delta_{2}>0$ such that $-\frac{\alpha \eta}{2}+\alpha V_{1}<-\frac{\alpha \eta}{4}$ for all $V_{1}<\delta_{2}$. Let

$$
\hat{K}_{\delta}=\left\{\left(T, T^{*}, V\right) \in K \cap U \mid T \geq \delta_{0}, V_{1} \geq \delta_{2}, T_{1}^{*} \geq \delta_{1} V_{1}\right\} .
$$

Consider $\left(T, T^{*}, V\right) \in(K \cap U) \backslash \hat{K}_{\delta}$ and let $\mu \in\left[0, \mu_{1}\right]$, then at least one of the following holds:

- $T<\delta_{0}$, in which case $\dot{W} \leq-L_{1}-\frac{\alpha \eta}{2}+L_{1} \leq-\frac{\alpha \eta}{2}$;

- $T_{1}^{*} / V_{1}<\delta_{1}$, in which case $\dot{W} \leq-L_{1}-\frac{\alpha \eta}{2}+L_{1} \leq-\frac{\alpha \eta}{2}$;

- $V_{1}<\delta_{2}$, in which case $\dot{W} \leq-\frac{\alpha \eta}{4} ;$

Hence, for all $\left(T, T^{*}, V\right) \in(K \cap U) \backslash \hat{K}_{\delta}$ and all $\mu \in\left[0, \mu_{1}\right]$, we have $\dot{W} \leq-\frac{\alpha \eta}{4}<0$. From this point forward, the proof is identical to the proof of Theorem 4 , so it will be omitted.

(b) Now we consider system (44). Let $W$ be the same as in part (a). Computing $\dot{W}$ for the system (44), we obtain

$$
\begin{aligned}
\dot{W}= & \left(N_{1}-1\right)\left[f^{\prime}(c)+\frac{k}{\gamma} f\left(\bar{T}_{1}\right)\right] \frac{\left(T-\bar{T}_{1}\right)^{2}}{T}+N_{1} \beta_{1} \bar{T}_{1}^{*}\left[3-\frac{V_{1} T \bar{T}_{1}^{*}}{\bar{V}_{1} \bar{T}_{1} T_{1}^{*}}-\frac{T_{1}^{*} \bar{V}_{1}}{\bar{T}_{1}^{*} V_{1}}-\frac{\bar{T}_{1}}{T}\right] \\
& -\left(N_{1}-1\right) \sum_{i=2}^{n} k_{i} V_{i}\left(\bar{T}_{i}-\bar{T}_{1}\right)+\mu \frac{V_{1}-\bar{V}_{1}}{V_{1}} \sum_{j=1}^{n} q_{1 j} N_{j} \beta_{j} T_{j}^{*}+\mu \sum_{i=2}^{n} \frac{N_{1}-1}{N_{i}-1} \sum_{j=1}^{n} q_{i j} N_{j} \beta_{j} T_{j}^{*},
\end{aligned}
$$

Recombining the terms, we find that

$$
\begin{aligned}
\dot{W}= & \left(N_{1}-1\right)\left[f^{\prime}(c)+\frac{k}{\gamma} f\left(\bar{T}_{1}\right)\right] \frac{\left(T-\bar{T}_{1}\right)^{2}}{T}+N_{1} \beta_{1} \bar{T}_{1}^{*}\left[3-\frac{V_{1} T \bar{T}_{1}^{*}}{\bar{V}_{1} \bar{T}_{1} T_{1}^{*}}-\frac{T_{1}^{*} \bar{V}_{1}}{\bar{T}_{1}^{*} V_{1}}-\frac{\bar{T}_{1}}{T}\right] \\
& -\left(N_{1}-1\right) \sum_{i=2}^{n} k_{i} V_{i}\left(\bar{T}_{i}-\bar{T}_{1}\right)+\mu \sum_{i=1}^{n} \frac{N_{1}-1}{N_{i}-1} \sum_{j=1}^{n} q_{i j} N_{j} \beta_{j} T_{j}^{*} \\
& -\mu q_{11} \frac{\bar{V}_{1} N_{1} \beta_{1} T_{1}^{*}}{V_{1}}-\mu \frac{\bar{V}_{1}}{V_{1}} \sum_{j=2}^{n} q_{1 j} N_{j} \beta_{j} T_{j}^{*},
\end{aligned}
$$

where the last term is clearly non-positive. Let

$$
\begin{aligned}
\alpha & =\left(N_{1}-1\right) \min _{i \geq 2} k_{i}\left(\bar{T}_{i}-\bar{T}_{1}\right)>0, \\
L & =\sup _{K} \sum_{i=1}^{n} \frac{N_{1}-1}{N_{i}-1} \sum_{j=1}^{n} q_{i j} N_{j} \beta_{j} T_{j}^{*} \geq 0 .
\end{aligned}
$$

By Lemma 9. there exist $\eta, \mu_{a}>0$ such that $1^{\prime} V(t)>\eta$ for all $\mu \in\left[0, \mu_{a}\right]$ and all sufficiently large $t$. Hence, by shifting time forward if necessary, we have the inequality

$$
\begin{aligned}
\dot{W} \leq & -\epsilon\left(N_{1}-1\right) \frac{\left(T-\bar{T}_{1}\right)^{2}}{T}+N_{1} \beta_{1} \bar{T}_{1}^{*}\left[3-\frac{V_{1} T \bar{T}_{1}^{*}}{\bar{V}_{1} \bar{T}_{1} T_{1}^{*}}-\frac{T_{1}^{*} \bar{V}_{1}}{\bar{T}_{1}^{*} V_{1}}-\frac{\bar{T}_{1}}{T}\right] \\
& -\alpha\left(\eta-V_{1}\right)+\mu L-\mu q_{11} \frac{\bar{V}_{1} N_{1} \beta_{1} T_{1}^{*}}{V_{1}}
\end{aligned}
$$

which holds in $K$ for all $\mu \in\left[0, \mu_{a}\right]$. We combine the second and the last terms to obtain

$$
\begin{aligned}
\dot{W} \leq & -\epsilon\left(N_{1}-1\right) \frac{\left(T-\bar{T}_{1}\right)^{2}}{T}+N_{1} \beta_{1} \bar{T}_{1}^{*}\left[3-\frac{V_{1} T \bar{T}_{1}^{*}}{\bar{V}_{1} \bar{T}_{1} T_{1}^{*}}-\left(1+q_{11} \mu\right) \frac{T_{1}^{*} \bar{V}_{1}}{\bar{T}_{1}^{*} V_{1}}-\frac{\bar{T}_{1}}{T}\right] \\
& -\alpha\left(\eta-V_{1}\right)+\mu L .
\end{aligned}
$$

Further, we rewrite the above inequality as

$$
\begin{aligned}
\dot{W} \leq & -\epsilon\left(N_{1}-1\right) \frac{\left(T-\bar{T}_{1}\right)^{2}}{T}+N_{1} \beta_{1} \bar{T}_{1}^{*}\left[3\left(1+q_{11} \mu\right)^{1 / 3}-\frac{V_{1} T \bar{T}_{1}^{*}}{\bar{V}_{1} \bar{T}_{1} T_{1}^{*}}-\left(1+q_{11} \mu\right) \frac{T_{1}^{*} \bar{V}_{1}}{\bar{T}_{1}^{*} V_{1}}-\frac{\bar{T}_{1}}{T}\right] \\
& -\alpha\left(\eta-V_{1}\right)+\mu L+3 N_{1} \beta_{1} \bar{T}_{1}^{*}\left[1-\left(1+q_{11} \mu\right)^{1 / 3}\right] .
\end{aligned}
$$


Let $\mu_{b}>0$ be such that for all $\mu \in\left[0, \mu_{b}\right]$,

$$
\left(1+q_{11} \mu\right) \in\left[\frac{1}{2}, 1\right], \quad-\alpha \eta+\mu L+3 N_{1} \beta_{1} \bar{T}_{1}^{*}\left[1-\left(1+q_{11} \mu\right)^{1 / 3}\right] \leq-\frac{\alpha \eta}{2} .
$$

Now we let $\mu_{1}=\min \left[\mu_{a}, \mu_{b}\right]$, so that for all $\mu \in\left[0, \mu_{1}\right]$ and all points in $K$,

$$
\begin{aligned}
\dot{W} \leq & -\epsilon\left(N_{1}-1\right) \frac{\left(T-\bar{T}_{1}\right)^{2}}{T}+N_{1} \beta_{1} \bar{T}_{1}^{*}\left[3\left(1+q_{11} \mu\right)^{1 / 3}-\frac{V_{1} T \bar{T}_{1}^{*}}{\bar{V}_{1} \bar{T}_{1} T_{1}^{*}}-\left(1+q_{11} \mu\right) \frac{T_{1}^{*} \bar{V}_{1}}{\bar{T}_{1}^{*} V_{1}}-\frac{\bar{T}_{1}}{T}\right] \\
& -\frac{\alpha \eta}{2}+\alpha V_{1} .
\end{aligned}
$$

From this point forward, the proof is identical to the proof of part (a), so it will be omitted.

Theorem 9. Let the assumptions of Lemma 8 hold, let $U$ be the set from Theorem 8 , and define

$$
U^{\prime}=\left\{\left(T, T^{*}, V\right) \in \mathbb{R}_{+}^{2 n+1} \mid T_{1}^{*}+V_{1}>0\right\} \supset U .
$$

Then there exist $\mu_{0}>0$ and a continuous map $E:\left[0, \mu_{0}\right] \rightarrow U$ such that

1. $E(0)=E_{1}$ (where $E_{1}$ is the same as in Lemma 8), and $E(\mu)$ is an equilibrium of (43) or of (44) for all $\mu \in\left[0, \mu_{0}\right]$;

2. For each $\mu \in\left[0, \mu_{0}\right], E(\mu)$ is a globally asymptotically stable equilibrium of (43) or of (44) in $U^{\prime}$.

Proof. The proof is similar to that of Theorem [5]

\section{References}

[1] P. De Leenheer, and H.L. Smith, Virus dynamics: a global analysis, SIAM J. Appl. Math. 64 (2003), 1313-1327.

[2] J. Hofbauer, and K. Sigmund, Evolutionary Games and Replicator Dynamics, Cambridge University Press, 1998.

[3] V. Hutson, A theorem on average Liapunov functions, Mh. Math. 98 (1984), 267-275.

[4] V. Hutson, and K. Schmitt, Permanence and the dynamics of biological systems, Math. Biosc. 111 (1992), 1-71.

[5] A. Iggidr, J.-C. Kamgang, G. Sallet, and J.-J. Tewa, Global analysis of new malaria intrahost models with a competitive exclusion principle, SIAM J. Appl. Math. 67 (2006), 260-278.

[6] A. Korobeinikov, Lyapunov functions and global properties for SEIR and SEIS epidemic models, IMA Math. Med. Biol. 21 (2004), 75-83.

[7] J.P. LaSalle, Stability theory for ordinary differential equations, J. Diff. Eqns. 4 (1968), 57-65.

[8] M.Y. Li, and J.S. Muldowney, Global stability for the SEIR model in epidemiology, Math. Biosc. 125 (1995), 155-164.

[9] M.A. Nowak, and R.M. May, Virus dynamics, Oxford University Press, New York, 2000.

[10] A.S. Perelson, D.E. Kirschner, and R. De Boer, Dynamics of HIV infection of CD4 ${ }^{+}$T cells, Math. Biosc. 114 (1993), 81-125.

[11] A.S. Perelson, and P.W. Nelson, Mathematical analysis of HIV-1 dynamics in vivo, SIAM Rev. 41 (1999), 3-44.

[12] L. Rong, Z. Feng, and A.S. Perelson, Emergence of HIV-1 drug resistance during antiretroviral treatment, preprint.

[13] H.L. Smith, and P. Waltman, The Theory of the Chemostat: Dynamics of Microbial Competition, Cambridge University Press, 1994. 
[14] H.L. Smith, and P. Waltman, Perturbation of a globally stable steady state, Proc. Amer. Math. Soc. 127 (1999), 447-453.

[15] H.R. Thieme, Persistence under relaxed point-dissipativity (with application to an endemic model), SIAM J. Math. Anal. 24 (1993), 407-435.

[16] L. Wang, and M.Y. Li, Mathematical analysis of the global dynamics of a model for HIV infection of $\mathrm{CD}^{+}{ }^{+} \mathrm{T}$ cells, Math. Biosc. 200 (2006), 44-57. 